\title{
UN'INDAGINE COMPARATISTICA SULLA CONFIGURAZIONE DEI REATI SESSUALI PER COLPA (GRAVE) SUI PROFILI DI CONSENSO DELLA VITTIMA
}

\author{
A COMPARATIVE RESEARCH ON THE CRIMINALIZATION OF (GROSS) NEGLIGENCE \\ WITH REGARD TO THE VICTIM'S CONSENT IN SEXUAL OFFENSES
}

\section{Matteo L. Mattheudakis}

\begin{abstract}
Dottore di ricerca in diritto penale nell'Università degli Studi di Parma. Laurea specialistica in Giurisprudenza presso l'Università di Bologna. Laurea triennale in Scienze giuridiche conseguita presso l'Università degli Studi di Bologna. Avvocato presso il Foro di Bologna. E-mail: matteo.mattheudakis@unibo.it
\end{abstract}

Convidado

RIASSUNTO: L'articolo sviluppa un'indagine comparatistica sui reati sessuali e perviene al riscontro di una significativa tendenza internazionale al superamento del modello coercitivo tradizionale basato su violenza e minaccia, in favore di incriminazioni incentrate semplicemente su profili di consenso della vittima. L'Autore analizza i due modelli principali di disciplina penale basata sul consenso, «no means no»e «(only) yes means yes», esprimendo preferenza per il primo, e spiega perché anche la colpa grave, in alternativa al dolo, possa rappresentare un criterio di colpevolezza adeguato a bilanciare gli interessi in gioco.

Palavras-chave: Reati sessuali; violenza; inaccia; consenso; colpa grave.

ABSTRACT: The paper carries out a comparative research on sexual offenses and shows a significant international trend towards overcoming the traditional model which defines the crime around the concepts of violence or threat instead of around the victim's consent. The Author examines the two main models based on consent, "no means no" and "(only) yes means yes", expressing preference for the "negative" one, and explains why even gross negligence, as an alternative to intent, can represent an adequate mens rea criterion to balance the interests at stake.

Keywords: Sexual offenses; violence; threat; consent; gross negligence

SUMÁRIO: 1. I limiti di una disciplina dei reati sessuali basata su violenza e minaccia - 2. «La Manada»: la recente casistica spagnola e il relativo contributo al dibattito sulla qualificazione giuridica del fatto - 3. Quale modello di incriminazione basato sul consenso? Le principali alternative: «no means no»; «(only) yes means yes»-4. L'opportunità di punire un errore colposo sul consenso: la convergenza di alcune indicazioni comparatistiche in favore della colpa grave -5 . Extrema ratio e proporzione sanzionatoria 


\section{I LIMITI DI UNA DISCIPLINA DEI REATI SESSUALI BASATA SU VIOLENZA E MINACCIA}

Uno sguardo comparatistico mostra una pronunciata tendenza a costruire i reati sessuali incentrandoli sull'elemento del consenso. Nell'ambito di tali normative, a prescindere dal linguaggio impiegato, non è affatto raro percepire, contestualmente, i segnali di un'apertura alla colpa, talvolta riconosciuta esplicitamente quale criterio di imputazione relativamente al carattere indesiderato degli atti sessuali. L'obiettivo di questo scritto è appunto quello di verificare l'opportunità di incentrare i reati sessuali, in particolare quelli che possono essere commessi nei confronti degli adulti ${ }^{1}$, su profili di consenso e comprendere se, per tali profili, possa essere soddisfacente un'apertura alla colpa quale criterio di imputazione. La tesi che si intende sostenere è che sia opportuno superare il modello coercitivo tradizionale, basato su violenza e minaccia, in favore di incriminazioni basate semplicemente sul consenso - rectius: sul dissenso - e che una colpa di grado elevato possa rappresentare, in affiancamento al dolo, un adeguato criterio di colpevolezza. Necessario, però, è che in ogni caso il trattamento sanzionatorio risulti proporzionato.

Nell'ordinamento italiano, quello a cui chi scrive appartiene e che verrà quindi spesso assunto come punto di riferimento per le considerazioni di questo articolo, la disciplina vigente la cui disposizione principale è quella dell'art. 609-bis c.p., rubricato «violenza sessuale» ${ }^{2}-$ è andata incontro a critiche analoghe a quelle che hanno indotto altri Paesi a modificare la loro strategia di tutela di beni giuridici che sono indubbiamente tra i più delicati, se non in assoluto quantomeno nell'economia dei delitti contro la persona.

Guardando all'assetto attuale, si può preliminarmente osservare che riserve sono state espresse rispetto all'opzione in favore di un modello unitario per reprimere condotte di gravità anche molto diversa. Come è noto, applicazioni giudiziali dell'art. 609-bis c.p. si sono registrate sia con riferimento a stupri che a baci "rubati" e toccamenti repentini ${ }^{3}$. L'estensione dell'area della tipicità finisce così per essere troppo ampia, rendendo poco soddisfacente la mera previsione di un'attenuante per i casi di minore gravità. Meglio sarebbe prevedere una o due fattispecie autonome ulteriori, come suggerito da tempo da parte della dottrina ${ }^{4}$, pervenendo così a una disciplina maggiormente calibrata in termini di tipicità e di proporzione sanzionatoria.

\footnotetext{
1 Per i reati sessuali che possono essere commessi solo nei confronti di soggetti di giovane età il consenso è normalmente irrilevante, in quanto si presume "viziato" o comunque non sufficiente di fronte al rischio di turbare il corretto sviluppo psico-fisico.

2 «Chiunque, con violenza o minaccia o mediante abuso di autorità, costringe taluno a compiere o subire atti sessuali è punito con la reclusione da sei a dodici anni.

Alla stessa pena soggiace chi induce taluno a compiere o subire atti sessuali:

1) abusando delle condizioni di inferiorità fisica o psichica della persona offesa al momento del fatto;

2) traendo in inganno la persona offesa per essersi il colpevole sostituito ad altra persona.

Nei casi di minore gravità la pena è diminuita in misura non eccedente i due terzi».

${ }^{3}$ Per un quadro piuttosto aggiornato sulla giurisprudenza, si veda, ad esempio, S.R. PALUMBIERI, Art. 609 bis, in A. CAdoppi, S. CANestrari, P. Veneziani, Codice penale commentato con dottrina e giurisprudenza, Torino, Giappichelli, 2018, in particolare pp. 2531 ss.

${ }^{4}$ La proposta fa originariamente riferimento soprattutto alla tipizzazione di una fattispecie di molestie sessuali, prevista nei lavori parlamentari relativi alla riforma del 1996, ma poi non confluita nella disciplina definitivamente approvata. Sul punto, ad esempio, A. CADOPPI, Il delitto di violenza sessuale (art. 609-bis c.p.), in A. CADOPPI, P. VENEZIANI, Elementi di diritto penale. Parte speciale. Vol. II. Tomo I, Milano, Wolters Kluwer-Cedam, 2017, pp. 22 e 23 (nel contesto di un confronto tra la nozione di «atti sessuali» e quella di «atti di libidine» prevista dalla disciplina precedente); inoltre, tra i non pochi, M. BERTOLINO, La riforma dei reati di violenza sessuale, in Studium Iuris, 1996, p. 404 ss., alla quale si deve pure la redazione del documento di sintesi sul punto dei contributi del «Gruppo di lavoro sui reati in materia sessuale» (composto anche dai Professori Giuliano Balbi e Alberto Cadoppi) dell'Associazione Italiana dei Professori di Diritto Penale: Verso la riforma dei reati sessuali. Parte I: I reati stricto sensu sessuali, in www.aipdp.it, p. 5, presentato al VII convegno nazionale della stessa Associazione - «Il diritto penale tra recenti modifiche e progetti di riforma»-tenutosi a Torino nei giorni 9 e 10 novembre 2018; F. MACRÌ, Verso un nuovo diritto

Revista de Direito Brasileira | Florianólopis, SC | v. 25 | n. 10 | p. 280-306 | Jan./Abr. 2020
} 
Ai fini della presente trattazione è però un altro l'aspetto che merita di essere privilegiato: la soluzione unitaria appena menzionata è insoddisfacente anche (se non soprattutto) poiché, sul piano della tipicità, rende di per sé insufficiente il compimento di atti sessuali sgraditi alla persona coinvolta, ricalcando essenzialmente il tradizionale modello coercitivo, che fa di violenza o minaccia fattori centrali e praticamente imprescindibili per buona parte della casistica di riferimento.

È ormai piuttosto matura, anche nel panorama internazionale, la critica a un modello punitivo del genere, che, se interpretato stricto sensu, rischia di lasciare priva di tutela quella che sembra potersi comunque definire una vittima ${ }^{5}$, mentre, se piegato alla bisogna, come avviene costantemente nell'ordinamento italiano, finisce per consentire la condanna di imputati - non importa se astrattamente meritevoli di pena - in violazione delle più essenziali istanze di legalità, in particolare tramite una lettura particolarmente estesa dei concetti di violenza e minaccia ${ }^{6}$.

Formulazioni normative come quella italiana vigente sulla violenza sessuale mostrano limiti significativi in alcuni casi in particolare. Tra questi vi è senz'altro quello in cui il comportamento della persona coinvolta è passivo e non caratterizzato da alcuna forma di resistenza diversa da quella eventualmente opposta verbalmente. In casi del genere, in cui può pure emergere con assoluta chiarezza il carattere sgradito degli atti sessuali compiuti, la vittima - come la si è definita anche poc'anzi, in termini non strettamente dipendenti dall'area della punibilità de iure condito - può tenere un atteggiamento apparentemente neutro o comunque non "combattivo" per diversi motivi: tra quelli che trovano più solido riscontro nella letteratura extrapenalistica, una paralizzante percezione di impotenza, specie in contesti isolati e in ragione di una netta differenza di forza fisica tra i soggetti; la supposizione - importa relativamente se fondata o meno - di poter subire "ulteriore" violenza (cioè diversa dall'imposizione strettamente sessuale); il non raro

penale sessuale. Diritto vivente, diritto comparato e prospettive di riforma, Firenze, Firenze University Press, 2010, pp. 44 ss., 201 e 202, che, al riguardo, parla anche di «atti sessuali minori». In termini problematici, a margine di una considerazione dei possibili inconvenienti della scelta di ricorrere a una circostanza attenuante per i «casi di minore gravità», P. PISA, Le nuove norme contro la violenza sessuale. Il commento, in Dir pen. proc., 1996, pp. 286 e 287. Sempre nell'ottica di rendere più proporzionate le fattispecie poste a tutela della libertà sessuale e le relative risposte sanzionatorie, si veda anche B. ROMANo, Proposte di riforma nei delitti contro la sfera sessuale della persona, in Dir. pen. cont., 29 novembre 2018, pp. 4-6, che, parlando esplicitamente di «"spacchettamento" del delitto di violenza sessuale», propone l'affiancamento a quest'ultimo, che perderebbe conseguentemente quote di tipicità, di un delitto di «abuso sessuale»; ma «sarebbe comunque opportuno l'inserimento nel nostro ordinamento di una norma specifica sulle molestie sessuali».

${ }^{5}$ Non sembrano fuori luogo considerazioni come quella, ad esempio, di M. VIRGILIO, Violenza sessuale e norma. Legislazioni penali a confronto, Ancona, Nuove Ricerche, 1996, p. 66, per cui rendere sussistente il reato «a condizione che l'aggressore abbia usata violenza e minaccia, significa imporre un onere di resistenza e dunque concedere tutela solo a chi dimostri di aver resistito. Questo equivale, in definitiva, a riconoscere la tutela penale solo a chi dimostri di aver "saputo meritare" quella tutela».

${ }^{6}$ In merito alla progressiva «volatilizzazione» del concetto di violenza nel contesto in esame, A. CADOPPI, Il delitto di violenza sessuale (art. 609-bis c.p.), cit., pp. 12 e 13. Si veda anche M. PAPA, La fisiognomica della condotta illecita nella struttura dei reati sessuali: appunti per una riflessione sulla crisi della tipicità, in Criminalia, 2018, in particolare pp. 217 ss., ove si parla di «smaterializzazione» di una fattispecie, quella di violenza sessuale, già in partenza tipizzata in modo non particolarmente pregnante semioticamente, a differenza di quella antecedente di violenza carnale, descritta come «esempio di condotta fisiognomicamente perfetta».

Più in generale, sull'alternativa tra «approccio "descrittivo"» e «approccio "normativo"» al concetto di violenza, usato quale vero e proprio banco di prova per testare la propria critica alle tesi più radicali nel declinare il divieto di analogia in materia penale, M. VogliotTI, Dove passa il confine? Sul divieto di analogia nel diritto penale, Torino, Giappichelli, 2011, pp. 91 ss., in esplicito "dialogo" con la contrapposta posizione di F. VigANò, La tutela penale della libertà individuale, I, L'offesa mediante violenza, Milano, Giuffrè, 2002, volta (quest'ultima) a ricavare «una nozione 'restrittiva' assai vicina al concetto sociale, pre-giuridico di violenza», che si collochi «entro limiti finalmente compatibili con il principio costituzionale di precisione» (p. 287). In senso critico sulle dilatazioni del concetto penalistico di violenza e in particolare su quello di «violenza impropria» di origine antoliseiana, si vedano anche G. DE SimONE, voce Violenza, in Enc. dir., XLVI, Milano, Giuffrè, 1993, pp. 881 ss.; M. MANTOVANI, voce Violenza privata, ivi, in particolare pp. $935 \mathrm{ss}$.

Revista de Direito Brasileira | Florianólopis, SC | v. 25 | n. 10 | p. 280-306 | Jan./Abr. 2020 
meccanismo autodifensivo di isolamento psicologico, una sorta di "dissociazione" mente-corpo proiettata a contenere l'intensità (almeno nell'immediato) del trauma ${ }^{7}$.

Le eventualità appena elencate sono destinate a riproporsi, in termini verosimilmente più accentuati, nei casi in cui siano più di uno i soggetti che indirizzano le proprie "attenzioni" sessuali alla vittima, rendendo pertinente l'assunzione quale fattispecie di riferimento di quella dell'art. 609-octies c.p. («Violenza sessuale di gruppo»), la quale però mutua parte significativa della tipicità, compresi i requisiti modali di coercizione qui criticati, dal prototipo dell'art. 609-bis c.p. ${ }^{8}$.

\section{2 «LA MANADA»: LA RECENTE CASISTICA SPAGNOLA E IL RELATIVO CONTRIBUTO AL DIBATTITO SULLA QUALIFICAZIONE GIURIDICA DEL FATTO}

Sui rapporti tra realizzazione plurisoggettiva di atti sessuali indesiderati e reazione della vittima, sembra eloquente ciò che è accaduto in Spagna negli ultimi anni. Ha fatto molto discutere l'opinione pubblica - ci sono state anche diverse manifestazioni di piazza - un caso avvenuto a Pamplona in una notte nel luglio del 2016, in occasione dei Sanfermines (i festeggiamenti di San Firmino): una ragazza di 18 anni ha seguito un gruppo di 5 uomini (di qualche anno più grandi) conosciuti poco prima, fino a quando la stessa è stata condotta in una zona appartata dell'ingresso di un palazzo e lì i 5 uomini hanno compiuto con lei atti sessuali di diversa natura ${ }^{9}$.

La mancanza di una significativa reazione fisica, in un certo senso - lo si preciserà a breve - confermata dalla versione della ragazza stessa, ha lasciato margine agli imputati per sostenere che i rapporti siano stati consensuali e che, solo in quanto abbandonata all'esito di questi fatti, la ragazza, per risentimento, abbia deciso di sporgere denuncia.

La ragazza, al contrario, ha raccontato di aver sì seguito i ragazzi senza costrizione inizialmente, ma di aver realizzato, a un certo punto, che le intenzioni degli uomini erano quelle di approfittarsi di lei sessualmente. A quel punto le è sembrato essere troppo tardi e si è sentita impotente, restando pietrificata in balia degli eventi. Accedendo a questa versione dei fatti, che l'atteggiamento della vittima possa essere interpretato come implicita manifestazione di consenso rispetto agli atti sessuali è quantomeno discutibile.

Uno dei momenti di maggior fermento dell'opinione pubblica ${ }^{10}$ si è avuto quando, il 20 marzo 2018, l'Audiencia Provincial de $\operatorname{Navarra}^{11}$ ha condannato a una pena detentiva di 9 anni gli imputati (solo) per abuso sexual (art. 181 c.p.) ${ }^{12}$, un reato punito (non certamente in modo lieve, ma) decisamente meno severamente rispetto alla agresión sexual (art. 178 c.p.), la cui maggior

\footnotetext{
${ }^{7}$ Tra i punti di riferimento della letteratura scientifica in questo senso, S.W. PorgES, La teoria polivagale. Fondamenti neurofisiologici delle emozioni, dell'attaccamento, della comunicazione e dell'autoregolazione, Roma, Fioriti, 2014, ove si possono leggere spiegazioni neuroscientifiche sulle reazioni della persona in situazioni di pericolo; reazioni che possono ben essere di carattere passivo, proprio come indicato nel testo.

${ }^{8} \mathrm{Si}$ veda, ad esempio, F. MAZZACUVA, La violenza sessuale di gruppo (art. 609-octies c.p.), in A. CADOPPI, P. VeneZIANI, Elementi di diritto penale. Parte speciale. Vol. II. Tomo I, cit., pp. 54 ss.

${ }^{9}$ La dottrina penalistica ha già dedicato diversi contributi al caso e alle sue implicazioni giuridiche. Tra questi, vi è persino un intero volume: P. FARAldo CABANA, M. AlCAlÉ SÁNCHEZ (directoras); S. RodríGUEZ LóPEZ, M.A. FUENTES LOUREIRO (coordinadoras), La Manada. Un antes y un después en la regulación de los delitos sexuales en España, Valencia, Tirant lo Blanch, 2018, ove, nelle pp. 13 ss., una ricostruzione dei fatti più accurata di quelle proposte dai vari organi di stampa (per la versione degli imputati, p. 16; per quella della vittima, p. 17).

${ }^{10}$ Lo testimonia la rassegna leggibile nella sezione «Reacciones a la sentencia de la Audiencia Provincial de Navarra» della ricca pagina Wikipedia specificamente dedicata al caso: https://es.wikipedia.org/wiki/Caso_de_La_Manada. Da lì, slogan come «Hermana, yo sí te creo» oppure «No es abuso, es violación».

${ }^{11}$ Audiencia Provincial de Navarra, Sec. II, 20 marzo 2018, n. 38, in www.lavanguardia.com, 26 aprile 2018.

${ }^{12}$ In tema, anche per un'istruttiva rassegna giurisprudenziale, E. PÉREZ ALONSO, Concepto de abuso sexual: contenido y límite mínimo del delito de abusos sexuales, in InDret, 3/2019.
}

Revista de Direito Brasileira | Florianólopis, SC | v. 25 | n. 10 | p. 280-306 | Jan./Abr. 2020 
gravità è essenzialmente determinata dall'impiego di violenza o minaccia: «utilizando violencia o intimidación» ${ }^{13}$.

Posto che, in ogni caso, è stata riconosciuta la mancanza di consenso della ragazza, anche perché, altrimenti, gli imputati sarebbero stati assolti, la (più "morbida") qualificazione giuridica della vicenda è evidentemente dipesa dalla mancanza di prova di una esplicita minaccia o di una violenza diversa dall'imposizione, di per sé, degli atti sessuali indesiderati. L'atteggiamento passivo della vittima, ripresa con il telefono e umiliata dal «branco» («La Manada»: così autodefinitosi in una chat WhatsApp), come le stesse immagini parrebbero dimostrare, era verosimilmente dovuto alla condizione di shock della ragazza e, una volta percepito un senso di ineluttabilità di ciò che stava accadendo, al tentativo più o meno conscio di "limitare i danni". Il contesto (di notte e in condizioni di relativo isolamento) e la oggettiva sproporzione di forza (pur solo potenziale) tra la ragazza (peraltro esile) e i 5 uomini era tale da far apparire vana qualsiasi opposizione, resa probabilmente ancora più difficile dalla previa assunzione di sostanze alcoliche.

Dopo una sostanziale conferma in secondo grado, nel 2019 il Tribunal Supremo ha ritenuto integrato il più grave reato di agresión sexual ${ }^{14}$. Per effetto di questa decisione, che ha ravvisato sussistente una «intimidación» e non un mero «prevalimiento» (cioè lo sfruttamento di un rapporto "sproporzionato" con la vittima), gli imputati hanno riportato una condanna a una pena detentiva di 15 anni.

Un fatto analogo è accaduto, sempre nel 2016, a Manresa, in Catalogna, in danno di una ragazza di 14 anni, abusata da 5 uomini ai quali non era stata opposta resistenza a causa dello stordimento dovuto a alcool e droghe. Pure in questo caso, che pare meno problematico dal punto di vista probatorio, l'assenza di necessità di ricorrere a esplicite violenze o minacce ha determinato la condanna a pene detentive tra i 10 e i 12 anni degli uomini per il reato di abuso sexual ${ }^{15}$, alimentando le proteste di buona parte dell'opinione pubblica ${ }^{16}$.

Anche in ragione di questi fatti, in Spagna, il dibattito sulla riforma dei reati sessuali è all'attenzione della politica ed è prevedibile che, come altrove, anche in questo Paese venga definitivamente abbandonato, quantomeno a livello di tipicità, il modello della coercizione, in favore di una disciplina incentrata sul consenso anche per i fatti più gravi. In questa direzione, appunto, si colloca un anteproyecto de ley orgánica approvato dal governo iberico all'inizio di marzo del 2020, che ambisce a ricondurre tutti i possibili attacchi alla libertà sessuale di una

13 Ampiamente sul punto, proprio a partire dal caso in commento, P. FARALDO CABANA, ¿Intimidación o prevalimiento? La sentencia de La Manada y los delitos sexuales en España, in Criminal Justice Network, 25 settembre 2018.

14 Tribunal Supremo, Sala de lo Penal, 4 luglio 2019, n. 344, consultabile all'indirizzo www.lavozdegalicia.es/default/2019/07/05/00171562321608473444967/Fichero/sentencia.pdf. Sui profili critici della della sentenza, J.A. LASCURAÍN SÁNCHEZ, La sentencia de La Manada: ¿todos contentos?, in Almacén de derecho, 4 novembre 2019.

15 Audiencia Provincial de Barcelona, Sec. XXII, 31 ottobre 2019, n. 813, consultabile all'indirizzo www.audiencias.vlex.es/vid/828174517. Per un commento critico, ad esempio, M. ACALE SÁNCHEZ, La miopia di 'vostro onore', in Criminal Justice Network, 10 novembre 2019.

${ }^{16} \mathrm{Si}$ veda l'editoriale di A. GARCIA, Consentimiento. Una nueva y polémica sentencia obliga a revisar el delito de violación, in https://elpais.com, 2 novembre 2019. Per un commento della vicenda nella prospettiva del giurista italiano, G.L. GATTA, Atti sessuali su minore incosciente per effetto dell'uso di alcool e droga: violenza o abuso sessuale? Il caso spagnolo della 'Manada de Manresa', nella prospettiva del penalista italiano. Sui confini dei concetti di violenza e minaccia, in Criminal Justice Network, 10 novembre 2019, il quale osserva come nell'ordinamento italiano il fatto sarebbe stato agevolmente - sembra però trascurato l'elemento costitutivo dell'induzione, dato forse un po' troppo per scontato e infatti mai menzionato - inquadrabile nell'ambito della fattispecie più grave, cioè quella di violenza sessuale (in questo caso di gruppo), la cui tipicità è integrata anche nelle ipotesi di abuso delle condizioni di inferiorità fisica o psichica della persona offesa (art. 609-bis, $2^{\circ}$ c., c.p., da intendersi richiamato anche nell'ambito dell'art. 609-octies c.p.). Sui rapporti tra atti sessuali compiuti nei confronti di una persona incosciente e harm principle, si veda l'interessante analisi di A. SPENA, Harmless Rapes? A False Problem for the Harm Principle, in Dir. \& quest. pubb., 2010, pp. 497 ss., che, condivisibilmente, ravvisa comunque una violazione del diritto all'autodeterminazione sessuale: «it is the violation of the victim's right to sexual autonomy» (p. 524).

Revista de Direito Brasileira | Florianólopis, SC | v. 25 | n. 10 | p. 280-306 | Jan./Abr. 2020 
persona non consenziente («cualquier acto que atente contra la libertad sexual de otra persona sin su consentimiento») all'ambito applicativo di un rinnovato art. 178 c.p., rubricato «agresión sexual» ${ }^{17}$.

L'esperienza spagnola induce a riflettere sull'importanza che possono avere, qui come in tanti altri casi, le Kulturnormen (norme di cultura), che, se devono concepirsi meno "pressanti" per il legislatore quando si pongono in chiave incriminatrice (o di inasprimento della punibilità), non per questo meritano di essere ignorate in prospettiva di riforma, pur non dovendo scadere nel "populismo penale"18. Con particolare riferimento al caso di Pamplona, l'opinione pubblica non ha accettato - sottinteso: una volta accreditata la versione della diciottenne - che un fatto percepito di gravità (almeno per quel settore) assoluta potesse non essere tale per il diritto positivo, o almeno per effetto di una sua interpretazione.

Nel panorama dottrinale italiano, anche chi ha proposto persino una tripartizione delle forme "principali" di reati sessuali, ha condivisibilmente ravvisato l'opportunità di una generale eliminazione del riferimento esplicito alla violenza e alla minaccia ${ }^{19}$. In tale ricostruzione, infatti, la differenza di gravità tra le proposte fattispecie di «stupro» e di «abuso sessuale» (a cui si affiancherebbe quella di «molestie sessuali») non è incentrata sulla presenza o meno di violenza o minaccia, ma, semplicemente, sulla diversità degli atti sessuali posti in essere nei diversi casi.

La pars destruens del modello adottato nell'ordinamento italiano può concludersi con un cenno alla casistica degli atti repentini e fugaci, che dimostra come la scelta di una fattispecie unica incentrata essenzialmente su violenza e minaccia riproponga gli stessi problemi di legalità pure nelle ipotesi di gravità più contenuta. Si tratta di quei casi in cui l'intrusione nella sfera sessuale altrui avviene in modo piuttosto insidioso, improvvisamente, e può persino cessare prima che venga accennata una reazione. La pressoché costante punizione ex art. 609-bis c.p. di questi comportamenti ha reso evidente, ancora una volta, l'appiattimento della violenza su ciò che di fatto si impone all'altra persona ${ }^{20}$.

A ben vedere, non sembra fuori luogo un confronto con la problematica emersa rispetto allo schema generale della violenza privata di cui all'art. 610 c.p., in particolar modo nei casi di trattamento sanitario c.d. arbitrario, in cui la fisiologica condizione di incoscienza nella quale può trovarsi il paziente soggetto a un intervento chirurgico ha talvolta posto le condizioni per variazioni non concordate (e magari nemmeno prospettate) del trattamento sanitario. Come è noto, lì si è registrata un'autorevole presa di posizione delle Sezioni unite della Cassazione nel senso della necessaria valorizzazione della dignità autonoma di ogni elemento costitutivo dell'illecito ${ }^{21}$.

\footnotetext{
${ }^{17}$ In termini critici sulla soluzione concepita dal governo spagnolo, J.A. LASCURAÍN SÁNCHEZ, Delitos sexuales: ¿una reforma progresista?, in Almacén de derecho, 28 marzo 2020, che, tra l'altro, lamenta il rischio di un appannamento di istanze di proporzione sanzionatoria per effetto di una ricostruzione unitaria del tipo, capace di attrarre fatti di gravità anche molto diversa: un profilo problematico che, come già rilevato nel testo, può essere riscontrato pure nella disciplina italiana vigente.

${ }^{18}$ Sull'argomento, sempre più trattato dalla letteratura penalistica, ad esempio, G. FIANDACA, Populismo politico $e$ populismo giudiziario; D. PUltTANò, Populismi e penale. Sulla attuale situazione spirituale della giustizia penale, entrambi in Criminalia, 2013, rispettivamente pp. 95 ss. e 123 ss.; M. DONINI, Populismo e ragione pubblica. Il postilluminismo penale tra lex e ius, Modena, Mucchi Editore, 2019; L. FerRAJOLI, Il populismo penale nell'età dei populismi politici, in Questione giustizia, 1/2019, pp. 79 ss.; G. INSOLERA, Il populismo penale, in disCrimen, 13 giugno 2019; E. AMATI, L'enigma penale. L'affermazione politica dei populismi nelle democrazie liberali, Torino, Giappichelli, 2020; S. AnAStasia, M. AnSElmi, D. FAlCinelli, Populismo penale: una prospettiva italiana, $2^{\mathrm{a}}$ ed., Milano, Wolters Kluwer, 2020. Proprio con riferimento alla sentenza dell'Audiencia Provincial de Navarra, si veda la profonda riflessione di M.A. PRESNO LINERA, ¿Son legítimas las sentencias juzgadas socialmente como injustas?, in Almacén de derecho, 6 novembre 2018.

${ }^{19}$ In questo senso, B. RomAno, Proposte di riforma nei delitti contro la sfera sessuale della persona, cit., pp. 4 ss., in particolare p. 5.

${ }^{20}$ Tra i contributi dedicati interamente al tema specifico, G. FRANCOLINI, Ai limiti della violenza sessuale: il dibattuto problema della rilevanza penale dei toccamenti repentini ed insidiosi, in Riv. pen., 2006, pp. 716 ss.

${ }^{21}$ Cass. pen., Sez. un., 18 dicembre 2008, n. 2437, (ad esempio) in Cass. pen., 2009, pp. 1793 ss., con commento di F. VIGANÒ, Omessa acquisizione del consenso informato del paziente e responsabilità penale del chirurgo: l'approdo

Revista de Direito Brasileira | Florianólopis, SC | v. 25 | n. 10 | p. 280-306 | Jan./Abr. 2020
} 
Le diverse soluzioni a cui perviene la giurisprudenza italiana in tema di violenza sessuale, più che potersi giustificare in ragione della differenza tra stato di incoscienza del soggetto passivo e repentinità dell'atto - in entrambi i casi il soggetto è esposto all'iniziativa altrui - sembrano piuttosto orientate da logiche di diritto penale d'autore: al giudicando sex offender, in quanto tipo di autore odioso, si riservano forzature in termini di legalità che il sanitario, il quale di base svolge una funzione fondamentale per la società, si ritiene non meriti.

\section{QUALE MODELLO DI INCRIMINAZIONE BASATO SUL CONSENSO? LE PRINCIPALI ALTERNATIVE: «NO MEANS NO»; «(ONLY) YES MEANS YES»}

Una volta percepita la necessità di superare il modello coercitivo a forma vincolata che caratterizza la disciplina italiana della violenza sessuale, si pone immediatamente la scelta dello specifico approccio per valorizzare il profilo del consenso della persona coinvolta nel compimento degli atti sessuali.

Il dibattito internazionale, recentemente intensificato dall'emergere di casi come quello del produttore di Hollywood Harvey Weinstein, alla base del più che noto «\#MeToo-movement» ${ }^{22}$, sta evidenziando come vi siano essenzialmente due alternative per punire gli atti sessuali indesiderati. Assumendo come punto di riferimento il consenso, si può individuare, in primo luogo, un modello, in una certa accezione, "negativo", che cioè subordina la punibilità al manifestarsi di un dissenso. L'alternativa è quella di ricorrere a un modello definibile "positivo", per il quale l'approccio sessuale può svilupparsi soltanto in presenza di una espressione di consenso; si parla, al riguardo, di «affirmative consent», che rende quindi non adeguata la presunzione del consenso stesso.

Anche per la loro universale intellegibilità nonché per la loro efficacia semantica, è bene richiamare le espressioni impiegate nella lingua inglese per descrivere sinteticamente i modelli appena accennati: «no means no»; «yes means yes», anche se per quest'ultimo sembra più fedele la versione per cui «(only) yes means yes», che meglio rende l'idea dell'imprescindibilità di una manifestazione di consenso quale condizione per la liceità del fatto ${ }^{23}$.

Una messa a fuoco delle differenze tra le due alternative può qui essere sviluppata soltanto per cenni e in termini funzionali a comprendere se si tratti, in ogni caso, di configurare una responsabilità dolosa oppure se, al contrario, l'imputazione colposa abbia margini per apparire plausibile in termini di politica criminale.

Il modello «no means no» rappresenta certamente un allontanamento rispetto alla tradizione delle legislazioni basate sulla violenza e la minaccia, ma fa comunque dipendere il reato sessuale da una sorta di resistenza, di opposizione della persona coinvolta: la differenza essenziale è che, secondo il modello «no means no», può ordinariamente - sono contemplate giustamente eccezioni per casi in cui la volontà è significativamente viziata - bastare una semplice opposizione verbale $^{24}$.

(provvisorio?) delle Sezioni unite, pp. 1811 ss., in particolare pp. 1813 ss. Sulla stessa sentenza, si vedano anche le note di G. FIANDACA, Luci ed ombre della pronuncia a sezioni unite sul trattamento medico-chirurgico arbitrario, in Foro it., 2009, II, cc. 306 ss.; S. TORDINI CAGLI, Profili penali del trattamento medico-chirurgico in assenza di consenso, in Resp. civ. prev., 2009, pp. 1060 ss., in particolare p. 1067.

${ }^{22}$ Per i riflessi sul diritto penale, per tutti, T. HöRNLE, \#MeToo - Implications for Criminal Law?, in Bergen Journal of Criminal Law and Criminal Justice, 2018, pp. 115 ss., in particolare pp. 122 ss.

${ }^{23}$ Per questa scelta terminologica, ad esempio, T. HöRNLE, \#MeToo - Implications for Criminal Law?, cit., pp. 129 ss. ${ }^{24}$ Nella prospettiva del modello "negativo" contemporaneo, il dissenso, in qualsiasi forma espresso, viene quindi preso sul serio e inteso sempre meno quale possibile forma di pudicizia imposta dal costume sociale per celare un desiderio erotico corrisposto. Conseguenza di ciò è che nessuna forma e nessun grado di violenza possono essere ordinariamente tollerati. Si va opportunamente sempre più lontano da quelle arcaiche e sessiste "logiche" riassunte con espressioni come vis grata puellae - in argomento, si veda, ad esempio, A. CADOPPI, Il delitto di violenza sessuale (art. 609-bis c.p.), cit., p. 13 - e, nell'area di common law, "“earnest resistance" or even resistance "to the utmost"》 (cioè seria o,

Revista de Direito Brasileira | Florianólopis, SC | v. 25 | n. 10 | p. 280-306 | Jan./Abr. 2020 
Il modello «(only) yes means yes» è quello più radicale e richiede sempre la manifestazione del consenso, non necessariamente attraverso parole, ma comunque in modo esplicito. Anche secondo questo modello, ci sono comunque dei casi di consenso viziato: in quei casi, «yes means no».

Negli Stati Uniti d'America, il modello basato su un «affirmative consent» ha avuto significative adesioni anche prima dell'affermarsi del \#MeToo-movement, in particolare nel contesto universitario: ad esempio, in California, nel 2014, sono state introdotte regole specifiche relative ai campus ${ }^{25}$.

Guardando alle riforme approvate in Europa più di recente, una disciplina che può essere senz'altro richiamata è quella tedesca risalente al 2016, che è stata incentrata sul consenso - una soluzione piuttosto innovativa rispetto all'intera area di civil law - e ha eliminato dalla fattispecie base del $\S 177$ il riferimento a violenza e minaccia, le quali oggi non sono più, quindi, requisiti essenziali della responsabilità penale, ma solo fattori di aumento di pena $(5)^{26}$. Ciò che conta è che vengano compiuti atti sessuali «contro la volontà riconoscibile di un'altra persona» («gegen den erkennbaren Willen einer anderen Person»).

Si è dunque accolto come modello di base quello per cui «no means no» (qui «Nein heißt Nein»), come conferma la dottrina tedesca ${ }^{27}$, mentre in casi eccezionali un assenso verbale o comunque esplicito - qui si riconoscono i tratti del diverso modello «(only) yes means yes» - è necessario, in particolare quando la persona coinvolta dagli atti sessuali sia considerevolmente limitata nella formazione o nell'espressione della volontà a causa del suo stato físico o mentale: così il $§ 177$ (2) 2. La punizione è inoltre garantita, per effetto del $§ 177$ (2) 1., nei casi in cui il soggetto destinatario delle "attenzioni”" sessuali dell'agente non sia in grado di maturare la propria contrarietà o di esprimerla. L'obiettivo evidente è quello di evitare che vi siano degli sfruttamenti delle possibili condizioni di vulnerabilità altrui.

Se è vero che la casistica degli atti sessuali repentini è difficilmente rapportabile a entrambi i modelli, in quanto si pone radicalmente al di fuori delle logiche comunicative degli stessi, pare opportuna, in linea di principio, la soluzione del sistema tedesco, che li punisce più o meno espressamente in via autonoma: il $§ 177$ (2) 3. si riferisce alle ipotesi in cui l'autore del fatto

addirittura, massima resistenza): S.J. SCHULHOFER, Unwanted Sex. The Culture of Intimidation and the Failure of the Law, Cambridge, London, Harvard University Press, 1998, pp. 19 e 20.

${ }^{25}$ In argomento, per un quadro piuttosto recente, E. GERSTMANN, Campus Sexual Assault. Constitutional Rights and Fundamental Fairness, Cambridge, Cambridge University Press, 2019, in particolare, pp. 141 ss., ove si tratta più da vicino il modello dell' «affirmative consent». Si veda anche, per un continuo aggiornamento, la sezione «the laws» del sito https://endrapeoncampus.org.

Alla base di questa particolare sensibilità rispetto agli ambienti universitari, oltre a dati statistici obiettivamente significativi - si veda un'analisi pubblicata dall'Association of American Universities il 3 settembre 2015: $A A U$ Climate Survey on Sexual Assault and Sexual Misconduct (2015), in www.aau.edu - vi sono senz'altro vicende giudiziarie come il caso statunitense Berkowitz, ambientato in un campus della Pennsylvania. L'imputato fu inizialmente condannato per rape, avendo praticamente costretto una ragazza a subire atti sessuali indesiderati, ma seguì un giudizio di segno diverso (con l'esclusione della responsabilità per rape), basato sul fatto che la vittima, la quale aveva dissentito verbalmente ma non aveva opposto alcun tipo di resistenza fisica, non fosse stata raggiunta da pressioni di natura violenta o minacciosa: in altre parole, fu ritenuta assente la necessaria «forcible compulsion». Per un approfondimento di questo caso, J. DrESSLER, S.P. GARVEY, Criminal Law. Cases and Materials, $7^{\text {th }}$ ed., Saint Paul, West Academic Publishing, 2016, pp. 454 ss.

${ }^{26}$ Per F. MACRÌ, La riforma dei reati sessuali in Germania. Centralità del dissenso e "tolleranza zero" verso le molestie sessuali tra diritto penale simbolico e potenziamento effettivo della tutela della sfera sessuale, in Dir. pen. cont., 24 novembre 2016, p. 27, le varie ipotesi tipizzate nel testo sarebbero «figure criminose aggravate (autonomamente, non prevedendo lo StGB, a differenza del Codice Rocco, il delitto circostanziato)». Non passa comunque inosservata la terminologia impiegata da T. HörnLE, The New German Law on Sexual Assault and Sexual Harassment, in German Law Journal, 2017, p. 1324, che parla di «aggravating circumstances». Per un commento aggiornato, si veda anche J. EISELE, § 177, in Schönke/Schröder Strafrechtgesetzbuch Kommentar, 30ª ed., München, Beck, 2019, pp. 1792 ss.

${ }^{27}$ T. HöRNLE, The New German Law on Sexual Assault and Sexual Harassment, cit., già a p. 1310.

Revista de Direito Brasileira | Florianólopis, SC | v. 25 | n. 10 | p. 280-306 | Jan./Abr. 2020 
specula sull'agire di sorpresa («der Täter ein Überraschungsmoment ausnutzt»), sembrando ricomprendere anche casi di maggiore gravità (atti sì repentini, ma di offensività più significativa rispetto a quelli meramente fugaci), giustificandosi forse così la parificazione sanzionatoria di partenza rispetto alle ipotesi ordinarie ${ }^{28}$.

$\mathrm{Si}$ aggiunge, qui, per inciso, che l'introduzione di un'analoga fattispecie ad hoc nell'ordinamento italiano dovrebbe essere congegnata in modo da applicarsi più che altro laddove sia, nella sostanza, presumibile il dissenso altrui, tenendo così conto di fattori come l'eventuale relazione intima (in corso) tra i soggetti coinvolti. Altrimenti, il rischio è che la tipicità del fatto si configuri pure in casi privi di reali esigenze protettive, lasciando al diritto penale il controverso compito di perseguire un'ambizione spiccatamente educativa, a prescindere e persino contro la volontà della supposta persona offesa, cosicché l'unica via d'uscita potrebbe essere la carenza del criterio di imputazione colpevole: un livello di selezione probabilmente troppo "tardo" nella sequenza logica degli elementi del reato, considerato anche il difficile raccordo con la scriminante del consenso dell'avente diritto, in quanto il consenso avrebbe comunque un ruolo essenziale già nell'economia dello stesso fatto tipico.

Pare interessante notare come la riforma d'oltralpe sia stata stimolata da due fattori in particolare: da un lato, la pressione di organizzazioni non governative e movimenti femministi; dall'altro lato, la necessità di dare attuazione alla Convenzione europea di Istanbul del 2011, che all'art. 36 indica agli Stati di incentrare la tutela della libertà sessuale sull'elemento del consenso della persona coinvolta ${ }^{29}$. La Convenzione di Istanbul è stata ratificata anche dall' Italia con la 1. 27 giugno 2013, n. 77, ma senza analoghe conseguenze per il momento.

Nel 2018, anche la Svezia (con norme entrate in vigore il $1^{\circ}$ luglio dello stesso anno) e l'Islanda (legge 5 aprile 2018, n. 16) hanno modificato la propria disciplina e un ruolo fondamentale è giocato anche qui dal consenso.

Nel testo codicistico svedese, nel kapitel $6, \S 1$, si punisce ora a titolo di stupro la realizzazione di un rapporto sessuale (o il compimento di atti sessuali di offensività comparabile) con una persona che non partecipa «volontariamente» («frivilligt»). La norma precisa che, nel valutare se una partecipazione sia volontaria o meno, occorre prestare particolare attenzione al fatto che il consenso sia stato espresso attraverso parole, azioni o in altro modo. Sono poi elencate delle ipotesi in cui deve ritenersi esclusa la partecipazione volontaria.

La altrettanto recente disciplina islandese ridisegna l'art. 194 del testo codicistico e incentra la tipicità sulla commissione di atti senza il consenso, precisando che il consenso è da considerarsi presente se espresso con «libera volontà» («frjálsum vilja»). Anche qui non mancano le ipotesi considerate in ogni caso non consensuali.

In entrambe queste riforme del 2018, l'apposizione dell'accento non solo sul consenso ma sulla sua espressione potrebbe rappresentare il segnale di un accoglimento del modello basato sull'affirmative consent, ma non si è mancato di rilevare qualche margine di ambiguità di tali discipline $^{30}$, per cui occorrerà attendere i primi assestamenti giurisprudenziali.

La matrice delle recenti riforme basate sul consenso, tra cui si può menzionare anche quella del 2019 del codice penale greco (art. 336, $5^{\circ}$ c.) ${ }^{31}$, al di là dei dettagli, sembra risalire a politiche criminali sviluppate in aree di common law. Rimanendo al contesto europeo, emblematica è la soluzione del Sexual Offences Act britannico del 2003, che, nelle sections da 1 a 4, sancisce che sussiste il fatto, rispettivamente, di «Rape», di «Assault by penetration», di «Sexual assault»e

\footnotetext{
${ }^{28}$ Nella dottrina italiana, diffusi riferimenti a queste ipotesi in F. MACRì, La riforma dei reati sessuali in Germania, cit., a partire da p. 3.

${ }^{29}$ T. HöRNLE, The New German Law on Sexual Assault and Sexual Harassment, cit., p. 1314.

${ }^{30}$ T. HÖRNLE, \#MeToo - Implications for Criminal Law?, cit., pp. 128 e 129, che riconosce, però, come la riforma svedese, al pari di quella tedesca, renda particolarmente importante l'aspetto comunicativo.

${ }^{31} \mathrm{Il}$ comma in questione attribuisce esplicita rilevanza agli atti sessuali realizzati «senza il consenso della vittima» ( «
}

Revista de Direito Brasileira | Florianólopis, SC | v. 25 | n. 10 | p. 280-306 | Jan./Abr. 2020 
di «Causing a person to engage in sexual activity without consent», quando la persona coinvolta nel compimento degli atti sessuali «does not consent», cioè «non acconsente». Richiedendosi che il soggetto acconsenta, potrebbe pensarsi alla necessità di un affirmative consent, ma la section 74 dello stesso corpo normativo, concepita con ruolo definitorio, sfuma sui connotati espressivi del consenso, concentrandosi più che altro sulla libertà dello stesso: «a person consents if he agrees by choice, and has the freedom and capacity to make that choice» ${ }^{32}$.

Nel documento dell'Associazione Italiana dei Professori di Diritto Penale presentato al Convegno nazionale di Torino del 9 novembre 2018, si riconosce che la tendenza internazionale è quella di incentrare la punizione dei sex offender sull'elemento del consenso ${ }^{33}$ e che pure la normativa italiana dovrebbe andare in quella direzione, anche se non ci si sofferma nel dettaglio sulla alternativa tra i due modelli sopra considerati ${ }^{34}$. Guardando alla disciplina penale oggi vigente nell'ordinamento italiano, seppur con una certa approssimazione, si può notare come il modello "negativo" sia stato impiegato nel contesto del delitto di violazione di domicilio (art. 614 c.p., $1^{\circ} \mathrm{e}$ $2^{\circ}$ c.: il soggetto attivo realizza il reato quando compie il fatto «contro la volontà espressa o tacita di chi ha il diritto di escluderlo»; «contro l'espressa volontà di chi ha il diritto di escluderlo»), mentre, quello "positivo" sembra essere stato posto alla base della recente tipizzazione della fattispecie di cui al $1^{\circ}$ c. dell'art. 612-ter c.p. («Diffusione illecita di immagini o video sessualmente espliciti»; tale $1^{\circ} \mathrm{c}$. si configura quando il fatto è commesso «senza il consenso delle persone rappresentate») $)^{35}$.

Tra le discipline di recente formulazione sul c.d. revenge porn, anche quella brasiliana del 2018 (l. n. 13.718) è incentrata sulla mancanza del consenso in termini simili a quella italiana: «sem o consentimento da vítima» (art. 218-C). Tra i reati di natura sessuale, una formula analoga si incontra anche nel testo dell'art. 215-A («Importunação sexual»), introdotto dalla stessa riforma del 2018: il fatto tipico si configura quando atti di libidine vengono commessi senza il consenso della persona coinvolta («sem a sua anuência»).

Tra gli aspetti più problematici di una disciplina incentrata sul consenso, vi è senz'altro la possibilità che anche una manifestazione di volontà, persino qualora del tutto esplicita, non sia ciò che appare: sono quei casi in cui si è portati a dire che «yes means no».

\footnotetext{
${ }^{32}$ Completa il quadro un catalogo di presunzioni sul consenso e sulla sua percezione, con onere probatorio ampiamente accollato all'imputato (sections 75 e 76). Uno sguardo d'insieme alle diverse disposizioni rivela di essere in presenza di una disciplina tra le più complesse e anche per questo non facilmente etichettabile, se non per il suo generale incentrarsi sul consenso. Al riguardo, si veda J. HERRING, Criminal Law, $10^{\text {th }}$ ed., London, Palgrave, 2017, p. 127, che sintetizza in questi termini: «The Sexual Offences Act has attempted to clarify the law on the meaning of consent, but the law is still very complex». L'impressione è confermata consultando l'ampia trattazione casistica sull'«assenza del consenso» e le relative presunzioni di P. RoOK, R. WARD, Sexual Offences. Law \& Practice, $5^{\text {th }}$ ed., London, Sweet \& Maxwell, 2016, pp. 73 ss., 117 ss. e (quanto al ragionevole convincimento sul consenso) 163 ss.

${ }^{33}$ In Europa, si pone in termini diversi la disciplina francese, che, nonostante sia stata modificata di recente, tramite la 1. 3 agosto 2018, n. 703, che ha inciso proprio sul fatto tipico di stupro («viol»: articolo 222-23 c.p.), non ha colto l'occasione per eliminare i riferimenti alle note coercitive del fatto, limitandosi ad allargare la tipicità della fattispecie, che ora si riferisce non solo al compimento di atti sessuali sulla vittima, ma anche all'imposizione alla stessa del compimento di atti sessuali sulla persona dell'autore. Per un aggiornato quadro della criminalità sessuale in Francia, in una prospettiva giuridica e sociologica, V. LE GoAZIOU, Viol. Que fait la justice?, Paris, Sciences Po, 2019.

${ }^{34}$ Verso la riforma dei reati sessuali. Parte I: I reati stricto sensu sessuali, cit., pp. 7 e 8 . Tra i contributi individuali dei partecipanti al gruppo di lavoro dell'Associazione Italiana dei Professori di Diritto Penale sul tema, G. BALBI, $I$ reati contro la libertà e l'autodeterminazione sessuale in una prospettiva di riforma, in Sist. pen., 3 marzo 2020, p. 2, che ribadisce l'opinione per cui sarebbe «più equilibrato, e rispondente a complessive istanze di razionalità sistematiche e di tutela, ritenere il fatto integrato dalla sola assenza di un valido consenso, non necessariamente esplicito, evidentemente, ma comunque attuale e riconoscibile».

${ }^{35} \mathrm{Nel}$ contesto di una trattazione di ampio respiro sul recente art. 612-ter c.p., richiama proprio quelle due fattispecie al fine di evidenziare le differenze di approccio nella tipizzazione di un reato incentrato su profili di consenso, G.M. CALETTI, Libertà e riservatezza sessuale all'epoca di Internet. L'art. 612-ter c.p. e l'incriminazione della pornografia non consensuale, in Riv. it. dir. proc. pen., 2019, pp. 2076 e 2077.
}

Revista de Direito Brasileira | Florianólopis, SC | v. 25 | n. 10 | p. 280-306 | Jan./Abr. 2020 
Il «\#MeToo-movement» ha acceso i riflettori sugli "abusi di potere" che non di rado si verificano negli ambienti lavorativi e che insegnano come l'accondiscendenza possa ben essere correlata alla possibilità, magari rimasta solo implicita, di condizionare, in positivo come in negativo, il percorso professionale. In questa casistica, le vicende finora considerate dalla cronaca sembrano dimostrare che le differenze d'accento possono essere talmente tante e così rilevanti da rendere assai difficile l'individuazione di criteri risolutivi preconfezionati. Ci si può giusto limitare a osservare che più il consenso è influenzato dalla prospettiva di evitare un danno ingiusto in un quadro di sopraffazione (e non meramente utilitaristico), più ci si avvicina al modello della minaccia così come inteso anche nell'ambito dei delitti contro la pubblica amministrazione ${ }^{36}$; in misura corrispondente, allora, il ricorso all'incriminazione si rende plausibile.

Pare discutibile la soluzione del legislatore brasiliano, che nel 2001 (con la legge 10.224) ha introdotto nel codice penale una fattispecie di «Assédio sexual» (art. 216-A), con la quale il problema dello sfruttamento della posizione lavorativa (così come l'abuso di autorità in genere) ha sì ricevuto esplicita considerazione, ma la modestia del trattamento sanzionatorio - è prevista una pena detentiva da uno a due anni - rispetto ad altre fattispecie sessuali lascia trasparire un poco condivisibile giudizio "pubblico" di lieve gravità degli abusi in ambito lavorativo 37 .

Altre logiche, evidentemente, orientano la soluzione di casi come quelli in cui la persona coinvolta nel compimento degli atti sessuali sia in condizioni di scarsa lucidità per aver assunto sostanze alcoliche o stupefacenti oppure presenti un deficit intellettivo.

36 È questa, pur con rilevanti precisazioni alla luce delle particolarità che la casistica può presentare, la prospettiva
della nota pronuncia delle Sezioni unite della Corte di cassazione italiana sulla distinzione tra concussione e induzione
indebita ex art. $319-q u a t e r$ c.p.: Cass. pen., Sez. un., 24 ottobre 2013 , n. 12228, (ad esempio) in Dir. pen. cont., 17
marzo 2014, con commento G.L. GATTA, Dalle Sezioni Unite il criterio per distinguere concussione e 'induzione
indebita': minaccia di un danno ingiusto vs. prospettazione di un vantaggio indebito, di cui si veda anche, per una più
diffusa illustrazione del concetto di minaccia da cui le Sezioni unite prendono evidentemente spunto, ID., La minaccia.
Contributo allo studio delle modalità della condotta penalmente rilevante, Roma, Aracne, 2013, in particolare pp. 149 ss. e 211 ss.

Sempre in merito alle relazioni sessuali collegate al luogo di lavoro, alcune grosse compagnie hanno adottato politiche aziendali molto restrittive, che in alcuni casi possono giustificare persino la sanzione di comportamenti che non sono molesti: nel novembre del 2019, il CEO di McDonald's è stato licenziato a causa di una relazione consensuale con una dipendente («consensual relationship with an employee»): https://edition.cnn.com/2019/11/03/business/mcdonaldsceo-steve-easterbrook-steps-down/index.html.

Oltre a una policy aziendale che considera «sexual harassment» un'ampia lista di comportamenti e che responsabilizza particolarmente i soggetti con funzioni direttive - «If you supervise or manage people, you must set a positive example by ensuring your conduct, including your employment decisions and workplace behaviors are free from discrimination and harassment» (https://corporate.mcdonalds.com/content/dam/gwscorp/scale-forgood/GP_Harassmentpolicy_0119_v3.pdf, p. 2) - la multinazionale ha attivato anche un sito internet per esplicitare la propria campagna contro le molestie sul luogo di lavoro e questo sito riprende, non causalmente, le parole chiave del «\#MeToo-movement»: https://metoomcdonalds.org.

Il caso del CEO di McDonald's è forse un chiaro esempio dei possibili effetti collaterali del «\#MeToo-movement», perché non manca il rischio che venga affermato il principio per cui una relazione sessuale tra persone che frequentano lo stesso ambiente lavorativo ma sono in posizione gerarchica diversa sia non solo un caso inopportuno - e, a ben vedere, può naturalmente non essere nemmeno tale - ma, necessariamente, anche un caso di consenso viziato. Il licenziamento non è formalmente una sanzione criminale e si basa su una violazione contrattuale, ma il rischio è che, nondimeno, si promuovano modelli presuntivi riguardo al consenso che anche il diritto (e il processo) penale potrebbe usare nei casi dubbi.

${ }^{37}$ In argomento, si veda, ad esempio, A.L. SABADELL, Forme di "patriarcalismo" giuridico-penale in Brasile. Il caso delle molestie sessuali e della violenza sessuale, in Criminalia, 2016, pp. 563 ss., in particolare pp. 566 ss., ove si può leggere una critica alla configurazione del reato in questione in termini tali da ricondurlo alla categoria dei «reati di minore gravità», con una serie di implicazioni non trascurabili sul piano pratico (ad esempio la pressoché automatica esclusione della pur minacciata pena detentiva). L'Autrice, che muove da una prospettiva dichiaratamente femminista, denuncia peraltro una diffusa ritrosia anche nella comunità dei giuristi ad accogliere l'incriminazione di certi abusi sessuali (come quello in ambito lavorativo), poiché la repressione apparirebbe in conflitto con una "cultura" ancora oggi piuttosto radicata.

Revista de Direito Brasileira | Florianólopis, SC | v. 25 | n. 10 | p. 280-306 | Jan./Abr. 2020 
Nei repertori di giurisprudenza italiana si trovano recenti massime che, se prese alla lettera, rischiano di ridurre significativamente la libertà sessuale "positiva"38 dei soggetti coinvolti quando almeno uno di questi si trovi in condizioni di ubriachezza: «In tema di violenza sessuale, posta l'annoverabilità dello stato di ubriachezza tra le condizioni di "inferiorità física o psichica" previste dall'art. 609 bis, comma 2, n. 1, c.p. e la conseguente invalidità del consenso eventualmente prestato da chi si trovi in detto stato (pur quando derivante da volontaria assunzione di alcolici) al compimento di atti sessuali, deve ritenersi che risponda del reato in questione il soggetto che, avendo cognizione dello stato di ubriachezza in cui si trova la persona offesa, la induca, abusando di tale stato, a compiere o a subire taluno di detti atti» ${ }^{39}$.

Al riguardo, sembrano condivisibili quelle discipline e quelle posizioni dottrinali più possibiliste sull'adeguatezza (riconosciuta anche dall'attuale normativa tedesca) di un affirmative consent nei casi di handicap e di ubriachezza ${ }^{40}$. Come spesso, anche in questo caso, approcci troppo drastici non sembrano condurre a soluzioni ragionevoli.

Quantomeno in una prospettiva de iure condendo, sembra opportuno considerare l'eventuale presenza di una strumentalizzazione, da parte dell'agente, della condizione di debolezza altrui, ma anche ricorrendo tale condizione non sembra da sottovalutare l'effettivo grado di capacità residua di esprimere un consenso che possa ancora definirsi tale e che potrebbe bilanciare la malizia e il cinismo dell'altra persona, la quale, peraltro, potrebbe anch'essa trovarsi in condizioni di alterazione, come può ben accadere: per intendersi, si pensi alla tutt'altro che rara assunzione comune di alcolici in un locale notturno, che, se anche fosse finalizzata a far allentare i freni inibitori dell'altra persona per poter così più facilmente allacciare con lei un contatto intimo, non dovrebbe automaticamente (cioè almeno fino a un certo livello di ubriachezza) comportare una radicale privazione della facoltà di autodeterminazione. Se è chiaro che tracciare una linea di confine è complesso, non va trascurato che una preclusione grossolana potrebbe persino penalizzare indebitamente la libertà sessuale "positiva" di quei soggetti che invece si vogliono tutelare $^{41}$. Per questo, soprattutto guardando alle ipotesi di occasionale alterazione da alcolici o stupefacenti, si potrebbe almeno pensare a una procedibilità a querela.

Gli aspetti appena considerati fanno emergere alcune delle difficoltà di tipizzazione dell'illecito facendo perno sulla volontà della persona coinvolta nel compimento di atti sessuali. Si tratta, a ben vedere, di questioni che rilevano già sul piano della tipicità - dell'actus reus nella prospettiva di common law - e che qui non sono ancora state vagliate accuratamente alla luce dei pertinenti profili di colpevolezza. L'approccio prescelto poggia semplicemente su ragioni di convenienza espositiva e non implica, ovviamente, che si debba pensare ai due livelli in termini completamente disgiunti, essendo più che evidenti le interrelazioni, sia sul piano sostanziale che probatorio. È così che, per avere una migliore visione di insieme, sembra opportuno tenere ora in

\footnotetext{
${ }^{38}$ Sul punto, si veda, ad esempio, A. CADOPPI, Il delitto di violenza sessuale (art. 609-bis c.p.), cit., pp. 9 e 10, che distingue tra libertà sessuale «in senso positivo»e «in senso negativo», in termini sostanzialmente corrispondenti alla distinzione giusfilosofica più generale tra «libertà positiva» (o «libertà di»; «freedom to») e «libertà negativa» (o «libertà da»; «freedom from»), su cui, per tutti, N. BoBBIO, voce Libertà, in Enciclopedia del Novecento, Roma, Treccani, 1978, (ora) in www.treccani.it.

${ }^{39}$ Cass. pen., Sez. III, 17 gennaio 2018, n. 38863, in DeJure.

${ }^{40}$ In questo senso, pronunciandosi sulla disciplina tedesca, T. HöRNLE, \#MeToo - Implications for Criminal Law?, cit., p. 131.

${ }^{41}$ Coglie appieno le difficoltà a cui ci si riferisce nel testo M. PAPA, Quando c'è l'ebrezza il consenso della vittima è sempre anomalo: $i$ limiti delle nostre leggi, in www.repubblica.it, 15 settembre 2017: «Una soluzione è quella di attribuire alla persona ubriaca lo stesso status del bambino, considerandola sessualmente intangibile. Al pari del pedofilo, chi la tocca è punito automaticamente. Questa soluzione assicura massima protezione alle potenziali vittime. Ma elimina radicalmente la libertà di dire sì: anche al partner abituale la sera di Capodanno; come Ulisse con le sirene, la persona che beve è legata al palo: per il suo bene, si dice. È però una soluzione eccessiva e non priva di venature paternalistiche. Bisognerebbe rafforzare la tutela della persona ubriaca, senza tuttavia privarla totalmente della capacità di decidere ed evitando di sancire un irrealistico monopolio del sesso sobrio».
}

Revista de Direito Brasileira | Florianólopis, SC | v. 25 | n. 10 | p. 280-306 | Jan./Abr. 2020 
sospeso il giudizio sui due modelli alternativi di valorizzazione del consenso, per arrivare a prendere posizione contestualmente all'analisi dei problemi di imputazione colpevole.

\section{L'OPPORTUNITÀ DI PUNIRE UN ERRORE COLPOSO SUL CONSENSO: LA CONVERGENZA DI ALCUNE INDICAZIONI COMPARATISTICHE IN FAVORE DELLA COLPA GRAVE}

Con riferimento a una disciplina come quella italiana sulla violenza sessuale, la questione dell'imputazione colposa dell'imposizione alla persona offesa di atti sessuali indesiderati è stata comprensibilmente trascurata, non solo in ragione della formale constatazione per cui attualmente sarebbe contra legem, ma anche perché violenza e minaccia sono condotte che si abbinano difficilmente a una colpevolezza diversa da quella dolosa: esse sono particolarmente evocative di un dolo che permea di sé il fatto tipico.

Nella prospettiva di pervenire a una nuova disciplina incentrata sul consenso, il quadro potrebbe però cambiare. Spogliando la fattispecie di violenza e minaccia, il problema della percezione della volontà altrui da parte dell'agente potrebbe risolversi, almeno in astratto, pensando anche all'imputazione colposa: quantomeno, si può dire che tale soluzione non incontrerebbe più ostacoli di carattere $\operatorname{logico}{ }^{42}$. Diventerebbe allora più che altro un problema di politica criminale, che ci si accinge qui ad affrontare.

Che non si tratti di uno scenario del tutto astratto è dimostrato da uno sguardo comparatistico, che indica che, quantomeno nell'area di common law, un'apertura alla responsabilità sostanzialmente colposa è non di rado prevista dalle discipline di settore o introdotta in via giurisprudenziale.

Si consideri, ad esempio, ancora il Sexual Offences Act britannico del 2003. Nelle già menzionate sections da 1 a 4 , non è solo previsto che la responsabilità penale operi se la persona coinvolta «does not consent»; ulteriore condizione è che «A [1'agente] does not reasonably believe that $\mathrm{B}$ [la vittima] consents».

La dottrina britannica ${ }^{43}$ testimonia che la regola è stata introdotta per evitare che si ripetessero giudizi ritenuti da molti insoddisfacenti come quello della House of Lords del 1975 nel noto caso Morgan $^{44}$. In tal caso, si era affermata la seguente regola: l'imputato per rape non deve essere condannato in presenza di un genuino convincimento («honest belief») sul consenso altrui, a prescindere dalla ragionevolezza di tale punto di vista. La questione qui si poneva perché il Royal Air Force Lieutenant Morgan aveva invitato alcuni colleghi a casa propria affinché consumassero rapporti sessuali con sua moglie, prospettando loro - così, almeno, è stato sostenuto dagli stessi uomini - che la donna si sarebbe opposta - e così fu - ma non perché non fosse davvero consenziente ma perché coltivava fantasie sullo stupro. La pronuncia della House of Lords ha comportato un cambiamento di prospettiva rispetto alla regola di common law valida fino a quel

\footnotetext{
${ }^{42}$ Nella dottrina italiana, si veda A. CADOPPI, Art. 609-bis c.p., in ID. (a cura di), Commentario delle norme contro la violenza sessuale e contro la pedofilia, $4^{\mathrm{a}}$ ed., Padova, Cedam, 2006, p. 540, che nota: «Non a caso il profilo dell'errore sul consenso si è manifestato con particolare rilievo pratico in quegli ordinamenti in cui i requisiti della violenza o della minaccia sono stati eliminati. In tali sistemi, il problema si è spostato sul versante soggettivo, sul piano, appunto, dell'errore sul consenso». Pur senza menzionare esplicitamente la colpa, l'Autore dà comunque conto del fatto che «in certi ordinamenti si è posto il problema se tale errore dovesse essere "ragionevole" per scusare».

${ }^{43}$ Ad esempio, K. STEVenson, A. DAVIES, M. GunN, Blackstone's Guide to The Sexual Offences Act 2003, Oxford, Oxford University Press, 2004, pp. 22 ss.

${ }^{44}$ House of Lords, 30 aprile 1975, [1975] UKHL 3; [1976] AC 182, in www.bailii.org. Dopo la sentenza della House of Lords, ma prima della riforma del 2003, si veda D.F. ALEXANDER, Twenty Years of Morgan: A Criticism of the Subjectivist View of Mens Rea and Rape in Great Britain, in Pace International Law Review, 1995, pp. 207 ss., che dà conto sia di casi precedenti - notando però, in essi, l'attribuzione di scarsa importanza ai profili di colpevolezza («Before the court's decision in Morgan, there was no established case law regarding the issue of mens rea in rape»: p. 211) - che degli sviluppi successivi.
}

Revista de Direito Brasileira | Florianólopis, SC | v. 25 | n. 10 | p. 280-306 | Jan./Abr. 2020 
momento - per cui era necessario un convincimento effettivo ma anche ragionevole («an honest and reasonable belief») sul consenso altrui - e che aveva determinato la condanna in primo grado degli imputati.

Taluno non ha mancato di sottolineare come la scelta legislativa attuale abbia evitato l'accertamento che normalmente si compie nelle ipotesi colpose, cioè il «reasonable man test», perché, a ben vedere, non si farebbe astrazione dalle caratteristiche dell'agente specifico, essendo attribuita esplicita rilevanza a tutte le circostanze del caso concreto ${ }^{45}$. In ognuna delle disposizioni sopra citate del Sexual Offences Act, subito dopo la descrizione dell'illecito vi è appunto la seguente precisazione: "Whether a belief is reasonable is to be determined having regard to all the circumstances, including any steps A has taken to ascertain whether B consents».

Autorevolmente, si è ritenuto comunque possibile riconoscere la natura colposa di una simile imputazione: «Absence of reasonable belief postulates an objective test, so that liability is based on negligence» ${ }^{46}$. In effetti, a prescindere dagli spesso fuorvianti aggettivi «oggettivo»e «soggettivo», il fatto che il giudizio sia arricchito anche dalle caratteristiche dell'agente concreto non può che avvicinarlo ancora di più alla colpa che non alla responsabilità oggettiva, almeno secondo il punto di vista del giurista di civil law contemporaneo, che concepisce sempre più la responsabilità colposa in termini individualizzati.

Guardando oltre oceano, una certa assonanza rispetto alla disciplina britannica si può riscontrare nell'assetto normativo del Canada, che prevede una regola simile in tema di errore sul consenso: tale errore può escludere la punibilità, ma non se il soggetto agente non ha adottato le misure ragionevoli per verificare il consenso altrui: «did not take reasonable steps [...] to ascertain that the complainant was consenting» (section 273.2) ${ }^{47}$.

La frammentata disciplina statunitense suggerisce di non entrare nei dettagli delle varie soluzioni $^{48}$, ma, piuttosto, di dare conto del fatto che la dottrina ha riscontrato nella maggior parte degli Stati il ricorso, quantomeno in via giurisprudenziale, (pure) a un'imputazione sostanzialmente $\operatorname{colposa}^{49}$, anche se il quadro potrebbe mutare a breve alla luce di un significativo percorso di

\footnotetext{
${ }^{45}$ In questo senso, ad esempio, P. ROOK, R. WARD, Sexual Offences, cit., in particolare, p. 163.

${ }^{46}$ R. CARD, A.A. GILlESPIE, M. HIRST, Sexual Offences, Bristol, Jordans, 2008, p. 32.

${ }^{47}$ In argomento, si veda, ad esempio, K. ROACH, Criminal Law, $7^{\text {th }}$ ed., Toronto, Irwin Law, 2018, pp. 484 ss., in particolare pp. 490 ss.

${ }^{48}$ Va giusto segnalata la peculiarità dello Stato più popoloso e certamente tra i più significativi sotto molti punti di vista, cioè la California, che nel suo penal code prevede un criterio di imputazione sostanzialmente colposo con riferimento più che altro alla capacità altrui di esprimere il consenso: così, ad esempio, già nella section 261 (1), dove la responsabilità per rape viene configurata (anche) nei casi in cui «a person is incapable, because of a mental disorder or developmental or physical disability, of giving legal consent, and this is known or reasonably should be known to the person committing the act».
}

${ }^{49} \mathrm{Si}$ vedano, ad esempio, H.F. Fradella, C. FAHMY, Rape and Related Offenses, in H.F. Fradella, J.M. SuMNER (edited by), Sex, Sexuality, Law, and (In)Justice, New York, Routledge, 2016, p. 150: «To avoid the problems with a subjective approach to the mens rea for rape, many U.S. states - indeed the majority of them - have opted for a negligence standard».

Si veda, inoltre, A.E. TASLITZ, Willfully Blinded: On Date Rape and Self-Deception, in Harvard Journal of Law \& Gender, 2005, pp. 381 ss. e, in particolare, p. 384 («Negligence is the mens rea requirement for rape liability imposed by the majority of American jurisdictions since the mid-1970s»), nonché p. 446, dove si conclude nel senso che un'imputazione sostanzialmente colposa (basata su canoni di ragionevolezza) rispetto all'elemento del consenso della persona con cui si compiono atti sessuali potrebbe rappresentare un'adeguata risposta almeno per la casistica di «selfdeception», cioè, letteralmente, di «auto-inganno» (meglio descritto a p. 382: «Self-deception occurs when the alleged rapist consciously, but incorrectly, believes that he has the woman's consent when, at some less-than-fully conscious level, he knows otherwise») rispetto all'effettività del consenso altrui, in particolare da parte dell'uomo nei confronti della donna: «male self-deception». In tali situazioni - l'articolo si concentra, in particolare, sulla vicenda che coinvolse il famoso giocatore di pallacanestro Kobe Bryant, sospettato dello stupro della dipendente di un albergo del Colorado nel 2003 - l'Autore ravvisa peraltro una maggior riprovevolezza rispetto ai casi ordinari di criminal negligence (che, in quel sistema giuridico, è comunque già una colpa di grado elevato: sul punto già nelle pp. 423 ss.) e che potrebbe rendere in qualche modo plausibili - così ritiene - le severe risposte sanzionatorie previste nel contesto dei reati sessuali. 
riforma dei reati sessuali del Model Penal Code intrapreso negli ultimi anni ${ }^{50}$. Andando nella direzione di elevare più esplicitamente a perno della disciplina modello il consenso, c'è da attendersi che gli Stati che ancora si basano su paradigmi vetusti come quello puramente coercitivo, un po' alla volta, si allineino alla prospettiva più moderna, peraltro sempre più gender-neutral. Dato che i lavori di riforma commissionati dall'American Law Institute sembrano individuare nella recklessness il criterio minimo di mens rea ${ }^{51}$, anche gli Stati che già incentrano la propria disciplina sul consenso ma che sono oggi aperti a un'imputazione (anche) colposa potrebbero avvertire l'esigenza di adeguarsi. In quest'ultimo caso, però, agli occhi del giurista di civil law, non si tratterebbe affatto di cancellare del tutto la rilevanza penale di un comportamento colposo: continuerebbero a essere punite forme di colpa grave purché "coscienti", cioè accompagnate dalla consapevole trascuratezza di un rischio piuttosto significativo. Lo stesso Model Penal Code, infatti, definisce il grado di rischio alla base tanto della negligence quanto della recklessness come «a substantial and unjustifiable risk»: section 2.02 (2).

Nell'area di civil law, tra i Paesi che stanno pianificando riforme, come la Spagna, si possono individuare posizioni dottrinali schierate apertamente a favore dell'introduzione di una responsabilità colposa rispetto al mancato apprezzamento del carattere indesiderato degli atti sessuali ${ }^{52}$.

Tra le riforme recentemente approvate, si può fare ancora una volta l'esempio di quella svedese del 2018. Nel $\S 1$ a e nel $\S 3$ del kapitel 6 del testo codicistico, risulta esplicitamente menzionata la colpa grave («grovt oaktsam») come ulteriore criterio di imputazione relativo alla partecipazione volontaria della persona coinvolta ${ }^{53}$. Un indubbio pregio di questa soluzione, se non

50 Nella dottrina penalistica statunitense, uno dei maggiori punti di riferimento della spinta riformatrice volta a valorizzare l'autonomia sessuale e, quindi, discipline incentrate sul consenso è l'opera di S.J. SCHULHOFER, Unwanted Sex, cit., pp. 99 ss. e 274 ss.; più di recente, ID., Reforming the Law of Rape, in Law \& Inequality: A Journal of Theory and Practice, 2017, pp. 335 ss. Lo stesso Schulhofer è stato recentemente incaricato, insieme a un'altra studiosa della New York University School of Law, Erin Murphy, di promuovere una riforma della parte del Model Penal Code dedicata ai reati sessuali (cioè l'articolo 213, composto di varie sections) che sia finalmente al passo coi tempi, mettendo al centro l'elemento del consenso: per l'ultima versione pubblicata al momento in cui si scrive, THE American Law Institute, Model Penal Code: Sexual Assault and Related Offenses, Tentative Draft No. 3 (April 6, 2017), Philadelphia, The American Law Institute, 2017. Nella manualistica recente, ne dà conto, ad esempio, J. DRESSLER, Understanding Criminal Law, 8ª ed., Durham, Carolina Academic Press, 2018, pp. 573 e 574.

A livello federale, lo United States Code prevede che i reati sessuali in esso disciplinati possano essere commessi «knowingly», cioè consapevolmente: Title 18 , sections 2241 ss.

${ }^{51}$ È da ritenersi che la scelta, pur concertata con i numerosi partecipanti al gruppo di lavoro, sia autorevolmente influenzata dal Project Reporter, emersa già in altre occasioni: risalendo indietro nel tempo, ad esempio, in S.J. SCHULHOFER, Taking Sexual Autonomy Seriously: Rape Law and beyond, in Law and Philosophy, 1992, p. 72, nota 95: «A separate question, that arises for any criminal prohibition, concerns liability in cases of mistake. Strict liability and negligence are arguably inappropriate standards of culpability in this context. But when the man knows that the woman means no (or is reckless about what she means), there is no legitimate reason to permit his conduct».

Sempre in una prospettiva di riforma, tra i vari contributi dottrinali, si distingue l'approccio "differenziato" di M.J. DunCAN, Sex Crimes and Sexual Miscues: The Need for a Clearer Line Between Forcible Rape and Nonconsensual Sex, in Wake Forest Law Review, 2007, in particolare pp. 1118 ss., la quale ritiene indispensabile quantomeno la recklessness per i casi più gravi, di forcible rape, mentre per i casi di nonconsensual sex (considerati dall'Autrice di rilevanza penale, ma degradabili anche a illecito civile) potrebbe essere adeguata anche un'imputazione per negligence. ${ }^{52}$ Così, ad esempio, J.A. LASCURAÍn SÁNCHEZ, ¿Qué es una violación?, in Criminal Justice Network, 2 ottobre 2018, nel paragrafo ¿Violación imprudente?: (dopo aver dato conto che l'errore sul consenso escluderebbe, de iure condito, la punibilità) «Esto, la impunidad de los delitos sexuales con esta clase de imprudencia, es lo que creo que, como han propuesto varios autores, debe remediarse con tipos específicos de agresiones imprudentes y abusos imprudentes. No hacerlo nos está llevando a dos consecuencias, a cuál peor: o bien la impunidad de conductas altamente reprobables, o bien el intento judicial de evitar lo anterior negando el error donde no se ha probado el conocimiento: afirmando tal conciencia sin la certeza que exige la constitucionalmente sagrada presunción de inocencia».

${ }^{53}$ La disciplina sulla responsabilità colposa (in particolare il $§ 1 \mathrm{a}$, che tipizza lo «stupro colposo», configurabile anche in casi di «gravità paragonabile al rapporto sessuale»; il $\S 3$ è invece dedicato all' «abuso sessuale colposo», riservato, in via residuale, agli altri atti sessuali) ha peraltro già trovato applicazione in un caso passato al vaglio della Corte suprema svedese (Högsta domstolen, 11 luglio 2019, caso B 1200-19). Un uomo e una donna si erano incontrati per

Revista de Direito Brasileira | Florianólopis, SC | v. 25 | n. 10 | p. 280-306 | Jan./Abr. 2020 
altro, è quello di aver dato rilevanza autonoma alle fattispecie colpose. Ciò, in generale, non è forse di per sé indispensabile, ma è importante l'implicazione di tale configurazione, perché il legislatore ha conseguentemente predisposto apposite cornici edittali tendenzialmente più tenui rispetto a quelle delle corrispondenti versioni dolose.

Arrivando a prendere posizione più esplicitamente sul quesito di fondo da cui è iniziato questo approfondimento, occorre innanzitutto esprimere delle riserve sulla possibilità di raggiungere un soddisfacente bilanciamento degli interessi in gioco associando l'imputazione colposa al modello che richiede un affirmative consent; ciò per diversi motivi, di seguito quantomeno accennati.

Come il modello «no means no», anche questo attribuisce un ruolo centrale a veri e propri profili comunicativi, ma la differenza è che configura una sorta di presunzione di dissenso di partenza, che può essere superata solo attraverso un accertamento preliminare delle intenzioni dell'altra persona: un vero e proprio obbligo cautelare sarebbe così imposto ab initio. Questo modo di concepire i rapporti umani a connotazione sessuale non sembra però pienamente aderente alla realtà e potrebbe spingere il diritto penale a colpire anche condotte dal disvalore quantomeno modesto, come si sta per dire.

Se 1"iter" implicitamente suggerito dal modello in esame può astrattamente apparire adeguato in termini di bon ton, già pensando concretamente ai primi "contatti" tra soggetti che, pur non essendo perfetti sconosciuti, non hanno particolare confidenza reciproca ${ }^{54}$, sembra proporre una prospettiva un po' forzata e rispetto alla quale potrebbe risultare necessario ottenere un esplicito consenso persino e, anzi, a maggior ragione, per il "primo bacio". Beninteso: non si vuole legittimare a ogni costo il bacio "rubato", ma è anche vero che dover necessariamente chiedere il permesso per scambiarselo, anche se non verbalmente - si lascia al lettore lo sforzo di immaginazione... - potrebbe, in alcuni contesti, rovinare l'atmosfera che altrimenti lo renderebbe più "naturale" oppure rappresentare persino un ostacolo psicologico per persone timide.

Volendosi realmente differenziare da quello basato sulla manifestazione del dissenso come i suoi sostenitori tengono in ogni occasione a ribadire, in alcuni casi parlando addirittura di «enthusiastic yes ${ }^{55}$ - il modello ora in considerazione potrebbe condurre all'assurdo di non

passare una serata insieme dopo aver avuto contatti solo via social network. I due avevano accettato di dormire nello stesso letto, ma la donna aveva espresso più volte segnali di "freddezza" circa la prospettiva di compiere atti sessuali. Nonostante ciò, l'uomo, a un certo punto, aveva inserito le proprie dita nella vagina della donna, registrando una reazione per lo più passiva di quest'ultima, che in ogni caso continuava a non dare segnali di volontaria partecipazione al compimento di atti sessuali. In primo grado e in appello, l'uomo era stato condannato per la versione sostanzialmente dolosa del reato di stupro, essendo stato ricostruito un approccio quantomeno di indifferenza sui profili di consenso della donna. La Corte suprema ha invece riqualificato il fatto, collocandolo nell'ambito applicativo della versione colposa, ravvisando sussistente un atteggiamento corrispondente alla colpa grave, che è appunto il criterio di imputazione introdotto con la riforma del 2018. Sul caso, si veda E. HofVERBERG, Sweden: Supreme Court Defines Negligent Rape, in Global Legal Monitor, 17 luglio 2019.

${ }^{54} \mathrm{Si}$ tratta di casi che nella dottrina di common law vengono ricondotti, nella prospettiva della loro possibile rilevanza penale, alla categoria della «date rape» (in cui l'accento è posto sulla conoscenza tra i due soggetti, infatti si ritiene che si tratti di una sottocategoria dell' «acquaintance rape», lo stupro tra conoscenti), su cui, ad esempio, A.E. TASLITZ, Willfully Blinded, cit., in particolare pp. 384 ss., riguardo ai rapporti tra tale casistica e la responsabilità colposa; inoltre, M.J. DunCAN, Sex Crimes and Sexual Miscues, cit., pp. 1087 ss. Nella dottrina italiana, si veda M. BERTOLINO, Libertà sessuale e tutela penale, Milano, Giuffrè, 1993, pp. 51 ss.

La categoria viene talvolta estesa fino a ricomprendere casi in cui il "corteggiatore" somministra alla vittima sostanze tali da farle perdere significativamente il controllo della situazione: allora si parla di «date rape drugs», che conferiscono però alla vicenda una connotazione difficilmente compatibile con la responsabilità colposa, perché, a ben vedere, tali pratiche vengono messe in atto proprio per aggirare il consenso altrui, evidentemente perché si sospetta che esso, in condizioni normali, non sarebbe presente.

${ }^{55}$ In argomento, ad esempio, M. COY, L. Kelly, F. VERA-GrAY, M. GARnER, A. KANYEREDZI, From 'no means no' to 'an enthusiastic yes': changing the discourse on sexual consent through Sex and Relationships Education, in V. Sundaram, H. SAUnders (edited by), Global Perspectives and Debates on Sex and Relationships Education. Addressing Issues of Gender, Sexuality, Plurality and Power, London, Palgrave Macmillan, 2016, pp. 84 ss.

Revista de Direito Brasileira | Florianólopis, SC | v. 25 | n. 10 | p. 280-306 | Jan./Abr. 2020 
concepire adeguato un lento e inequivoco avvicinamento al corpo dell'altra persona, che pure le consentirebbe di esprimersi. Di fronte a una dinamica del genere, si potrebbe arrivare a ritenere che la persona rimasta impassibile non abbia affermato il proprio consenso e che, quindi, a prescindere dal suo effettivo gradimento dell'iniziativa, sia integrata la tipicità del fatto. Evidente il cortocircuito per cui lo spettro della tutela penale verrebbe così ad affacciarsi persino contro la volontà della persona la cui libertà sessuale si dovrebbe proteggere ${ }^{56}$.

Tale proceduralizzazione delle dinamiche sessuali, di matrice radicalmente femminista ${ }^{57}$, per quanto muova da intenti certamente comprensibili a fronte di una ancora eccessiva esposizione femminile ad abusi sessuali di varia natura, stenta però a tradursi in regole ragionevoli, a maggior ragione rispetto alla ben possibile evoluzione dei rapporti sessuali, perché richiede sostanzialmente un aggiornamento (anch'esso in forma espressa) del consenso in occasione di ogni passaggio al compimento di atti sessuali in qualche misura differenti rispetto a quelli già acconsentiti. Non sembra proprio questo il modo migliore per presidiare penalmente il (di per sé condivisibile) principio generale per cui il consenso deve persistere in ogni momento della relazione intima, non essendo sufficiente averlo "ricevuto" inizialmente a fronte dell'emergere, a un certo punto, di una volontà di segno diverso ${ }^{58}$.

\footnotetext{
${ }^{56} \mathrm{~A}$ tale riguardo, non sembra da sottovalutare nemmeno la possibile configurazione del reato - così dovrebbe essere, a rigor di logica - anche nei casi di approccio azzardato ma a un certo punto (comunque ex post) "coperto" dal consenso della vittima. Come dovrebbe essere inquadrata questa "degradazione" del ravvisato disvalore iniziale? La domanda non sembra banale, perché potrebbero isolarsi normativamente due segmenti comportamentali: quello "scoperto" dal punto di vista del necessario consenso e quello successivo in cui gli atti sessuali sono consensuali. A ben vedere, lo stesso problema può porsi anche rispetto agli altri più comuni modelli di incriminazione, compreso persino quello coercitivo tradizionale, anche se forse in minor misura e comunque con più riserve.

Nella direzione - certo non l'unica possibile - di pervenire a un'esclusione della responsabilità penale, gli strumenti tecnici posso essere diversi: ad esempio, si potrebbe pensare a una causa di non punibilità sopravvenuta oppure, volendo lasciare impregiudicata la punibilità, si potrebbe ipotizzare di "risolvere" la questione sul piano del regime di procedibilità, ma mettendo in conto il rischio di possibili strumentalizzazioni. In ogni caso, è dubitabile che un testo normativo prenda posizione su questa spinosa casistica, perché si esporrebbe probabilmente all'obiezione di calarsi in una prospettiva sessista, di riabilitazione implicita di paradigmi arcaici come quello (in realtà qui non del tutto pertinente) della vis grata puellae. Eppure il problema può ben fondatamente porsi e in dinamiche molto meno artificiose di quanto si possa magari pensare a prima vista: un esempio può essere quello della persona che, inizialmente, non acconsente all'approccio "tangibile" altrui, non per mancanza di attrazione reciproca, ma per l'impegno sentimentale con un'altra persona, finendo però per cedere alle avances $\mathrm{e}$, da lì in poi, partecipando a tutti gli effetti al compimento di atti sessuali. In casi del genere, non sembra così pruriginoso riconoscere tanto la genuinità del dissenso iniziale (o del mancato consenso) quanto quella del consenso sopravvenuto, quindi l'esigenza di ricercare una soluzione equilibrata non può lasciare indifferente il giurista.

Qui non vi è lo spazio per andare molto oltre, ma si consenta comunque di affermare che, almeno nei casi appena richiamati a titolo di esempio - e che non vanno affatto confusi con quelli in cui, a un vero e proprio abuso sessuale, specie in ambito domestico, segua, ma dopo un apprezzabile lasso temporale, una riappacificazione tra i soggetti e la ripresa di rapporti sessuali consensuali: nessuna ambiguità può essere lasciata rispetto allo spettro di famigerati istituti come la marital rape exemption (su cui, nella dottrina italiana, in particolare, A. SZEGÖ, Quando lo stupro è legale: la "marital rape exemption", in Riv. it. dir. proc. pen., 1995, pp. 853 ss.) - la punibilità non dovrebbe ordinariamente essere consentita.

${ }^{57}$ In alcuni contesti occidentali maggiormente che in altri la cultura femminista ha trovato nella comunità giuridica esponenti di primo piano che hanno catalizzato l'attenzione del dibattito scientifico su temi delicati come le prevaricazioni sessuali nei confronti delle donne. Si pensi al contributo della giurista nordamericana Catharine A. MacKinnon, tra le cui opere più influenti vi è senz'altro C.A. MACKINNON, Sexual Harassment of Working Women. A Case of Sex Discrimination, Hew Haven, London, Yale University Press, 1979. Sul percorso di questa corrente culturale - recentemente rappresentata, ad esempio, in U. ANDERSSON, M. EDGREN, L. KARLSSON, G. NILSSON (edited by), Rape Narratives in Motion, Cham, Palgrave Macmillan, 2019, utile anche per una comprensione dei presupposti della riforma svedese del 2018, in particolare nelle pp. 43 ss., 101 ss., 119 ss. e 223 ss. - autorevolmente, S.J. SCHULHOFER, The Feminist Challenge in Criminal Law, in University of Pennsylvania Law Review, 1995, pp. 2151 ss., in particolare, pp. 2170 ss., ove un ampio confronto con l'approccio femminista al tema della violenza sessuale.

${ }^{58} \mathrm{E}$ un principio giustamente affermato più volte anche dalla giurisprudenza italiana e che ha peraltro ricevuto diverse declinazioni, come quella di Cass. pen., Sez. III, 18 marzo 2015, n. 9221, in Guida dir., 2016, 17, p. 62, così massimata: «Il comportamento di chi porti a conclusione un rapporto sessuale, inizialmente voluto dal partner, ma proseguito con Revista de Direito Brasileira | Florianólopis, SC | v. 25 | n. 10 | p. 280-306 | Jan./Abr. 2020
} 
Se così è, allora, tale modello rivela un'impronta letteralmente educativa alla sessualità, di sua tendenziale omologazione, quantomeno sul piano comunicativo, col rischio però di affidare al diritto penale una funzione promozionale che, tanto più in un settore così delicato, non sembra competergli ${ }^{59}$.

Per ciò che qui più interessa, poi, non è nemmeno possibile escludere che una simile proceduralizzazione comporti una sorta di fagocitazione della colpa da parte del dolo, analogamente a quanto avviene con riferimento a non pochi reati omissivi propri ${ }^{60}$. Si intende dire che la fisionomia anfibia della fattispecie incriminatrice potrebbe rendere direttamente doloso l'illecito commesso da chi ometta - e per ciò solo - di procurarsi un assenso preventivo al compimento degli atti sessuali. Nonostante la decisa precauzionalizzazione di fondo dell'intero contesto, che di fatto determina una sorta di immanenza dell'Anla $\beta$, la responsabilità colposa finirebbe per potersi configurare nei soli casi di erronea percezione circa la (in realtà non avvenuta o non genuina) manifestazione del consenso ${ }^{61}$.

Oltre a essere più convincente in generale, dovendosi quindi condividere l'approccio della riforma tedesca che lo ha chiaramente elevato a canone di incriminazione ordinario, il modello «no means no» è anche quello che meglio si combina con un'imputazione colposa. Si tratterebbe, in altre parole, di concepire un addebito colpevole (anche) per non aver riconosciuto (non già un elemento - per così dire - negativo e poco tangibile, quale il mancato consenso, ma) il dissenso in qualche modo manifestato dalla persona offesa.

Quanto alla fisionomia del parametro di imputazione, se è vero che è molto difficile rinvenire un vero e proprio agente modello in questo settore, anche perché sembra una forzatura la prospettiva di una rigida canonizzazione delle dinamiche interpersonali intime, un certo livello di oggettivizzazione del giudizio pare comunque indispensabile, come in tutte le ipotesi di colpa. Il criterio selettivo che sembra meno esposto al rischio di permettere una sussunzione di casi di speciale ambiguità sul consenso è quello della colpa grave. Al riguardo, sembra allora sostanzialmente convincente la scelta di quegli ordinamenti - si pensi, per restare nell'ambiente di civil law, a quello svedese - che richiedono una colpa, anche incosciente, ma di grado elevato, definibile, qui, segnatamente in termini di significativa riconoscibilità del dissenso altrui.

In caso di marchiano travisamento o di grossolana noncuranza dei segnali di manifestazione del dissenso della vittima, più che avventurarsi nelle paludose retoriche del dolo eventuale, sembra accettabile un'imputazione normativa che non dipenda esclusivamente dal punto di vista dell'agente, il quale, altrimenti, potrebbe dimostrarsi talmente insensibile alla dimensione relazionale del compimento degli atti sessuali al punto da rendere credibile il suo effettivo convincimento di non aver incontrato alcun reale dissenso dell'altra persona. Di fronte a casi del genere - pare - non si tratterebbe di uniformare rigidamente l'approccio alla sessualità della collettività, ma, impregiudicata una (pur assai delicata) valutazione sulla misura soggettiva della colpa, di riaffermare quel minimo di civiltà che non dipende certo da sfumature percepibili e decifrabili solo da esperti in psicologia.

modalità sgradite o non accettate da quest'ultimo, integra il reato di violenza sessuale (fattispecie in cui la Corte ha ritenuto ravvisabile il reato nel comportamento di chi, secondo la prospettazione accusatoria, contro la volontà della donna, aveva consumato il rapporto sessuale con l'eiaculazione in vagina, non condivisa dalla donna)». In argomento, tra gli scritti più interessanti, M.C. PARMIGGIANI, Rape by omission, ovvero lo "stupro omissivo": note a margine di un recente caso californiano, in Ind. pen., 2005, pp. 311 ss.

59 Sulle criticità che possono ravvisarsi in sistemi liberali nell'assegnazione al diritto penale di una funzione promozionale, ampiamente, A. CADOPPI, Il reato omissivo proprio, I: profili introduttivi e politico criminali, Padova, Cedam, 1988, pp. 373 ss.; già prima, F. BRICOLA, Funzione promozionale, tecnica premiale e diritto penale, in Quest. crim., 1981, pp. 445 ss.

${ }^{60}$ Per tutti, A. CADOPPI, Il reato omissivo proprio, II: profili dogmatici, comparatistici e de lege ferenda, Padova, Cedam, 1988, pp. 933 ss.

${ }^{61}$ In argomento, ampiamente, K.K. FERZAN, Consent, Culpability, and the Law of Rape, in Ohio State Journal of Criminal Law, 2016, in particolare, pp. 417 ss. (e 433), ove pure una denuncia dei rischi di scivolamento verso la responsabilità oggettiva a prescindere dal titolo formale di responsabilità.

Revista de Direito Brasileira | Florianólopis, SC | v. 25 | n. 10 | p. 280-306 | Jan./Abr. 2020 
Se non si accettasse l'imposizione di alcun punto di vista oggettivo dell'ordinamento, allora l'argomentazione potrebbe spingersi a sindacare persino lo stesso ricorso al diritto penale per proteggere la libertà sessuale. È evidente che un simile esito non sarebbe accolto praticamente da nessuno. Del resto, la lezione storico-comparatistica insegna che la repressione degli abusi sessuali, pur con alcune differenze d'impostazione derivanti soprattutto dalla definizione del bene giuridico, è una delle poche vere costanti del diritto penale ${ }^{62}$, nonostante ciò conviva con una più che significativa cifra di casi non denunciati, anche alla luce del fatto che le statistiche indicano chiaramente che si tratta spesso di vicende tra "partner", smentendo lo stereotipo che pretende di dipingerlo come un fenomeno criminologico prevalentemente di strada ${ }^{63}$.

La ricerca del punto di equilibrio tra prevenzione generale e colpevolezza nella prospettiva fin qui tracciata deve coerentemente indurre a prendere le distanze da approcci che pretendano di ottenere un perfetto e indefettibile allineamento tra compressione della libertà sessuale e responsabilità penale. Per quanto magari suggestiva, soprattutto per il linguaggio impiegato, è fallace, perché prova troppo, la prospettiva femminista, riconducibile particolarmente a Catharine MacKinnon, per cui una tutela penale attenta al punto di vista dell'agente, consistente nel fornirgli una reasonable mistake defense, implicherebbe sostanzialmente l'inaccettabile risultato di considerare una «donna stuprata, ma non da uno stupratore» («a woman raped but not by a rapist» $)^{64}$. A tale considerazione si è condivisibilmente replicato che, nell'intero diritto penale, si possono fare moltissimi esempi tutt'altro che scandalosi di danno alla persona prodotto da altri, $\mathrm{i}$ quali, però, pur avendo magari integrato la tipicità della fattispecie, non sono punibili per difetto della necessaria colpevolezza ${ }^{65}$. Ciò vale non solo nel contesto giuridico in cui la MacKinnon ha lanciato la sua provocazione, ma, certamente, anche in ordinamenti come quello italiano, dove, altrimenti, si cancellerebbe il filtro selettivo e di garanzia della colpevolezza, trasformando il reato in un illecito di danno a forma talmente libera da spingerlo persino oltre gli orizzonti dell'illecito civile.

\section{EXTREMA RATIO E PROPORZIONE SANZIONATORIA}

La questione della proporzione sanzionatoria dell'imputazione colposa dei profili di consenso è un aspetto centrale nel dibattito dei Paesi di common law. Lì, in particolare nel sistema statunitense, rispetto a quello che si vuole qui proporre, si è spesso sviluppato ed è in via di affermazione un ragionamento in una certa misura diverso, forse un po' più di extrema ratio,

\footnotetext{
${ }^{62}$ Tra coloro che ne danno conto, F. MACRÌ, Verso un nuovo diritto penale sessuale, cit., p. 1. Si veda inoltre L. GoISIS, La violenza sessuale: profili storici e criminologici. Una storia 'di genere', in Dir. pen. cont., 31 ottobre 2012, in particolare pp. 4 ss.

${ }^{63}$ Già da diversi decenni, si denuncia un frequente vizio di prospettiva nell'analisi dei reati sessuali. Tra i contributi meno recenti, C.E. LEGRAND, Rape and Rape Laws: Sexism in Society and Law, in California Law Review, 1973, p. 926: «Rape laws, and legal discussions of those laws, are also shaped by the fear of the stereotypical maniac rapist who leaps from the bush with knife in hand».

Nel Rapporto SDGs 2018 pubblicato nel sito dell'Istituto Nazionale di Statistica (www.istat.it), si dà conto del fatto che «il 62,7\% degli stupri subiti nel corso della vita è stato commesso da un partner attuale o precedente». Per indicazioni dettagliate sulle assai basse percentuali di denuncia, si veda la sezione La consapevolezza e l'uscita dalla violenza dello stesso sito. In dottrina, in argomento, L. GoIsis, La violenza sessuale, cit., pp. 1 ss., in particolare p. 7.

${ }^{64}$ C.A. MACKInNon, Feminism, Marxism, Method, and the State: Toward Feminist Jurisprudence, in Signs, 1983, p. 654.

${ }^{65}$ Ad esempio, M. Vitiello, Punishing Sex Offenders: When Good Intentions Go Bad, in Arizona State Law Journal, 2008, pp. 662 e 663, ove una efficace critica proprio alla posizione dell'Autrice menzionata nel testo: «not all deaths caused by others are actionable under the criminal law. Even beyond accidental deaths, absent some kind of criminal negligence, beyond tort negligence, an actor is not guilty of involuntary manslaughter or negligent homicide» (p. 663). Sulla questione, si veda anche D. HUSAK, The Philosophy of Criminal Law. Selected Essays, Oxford, Oxford University Press, 2010, pp. 233 ss.
}

Revista de Direito Brasileira | Florianólopis, SC | v. 25 | n. 10 | p. 280-306 | Jan./Abr. 2020 
almeno apparentemente, ma non per questo da considerarsi necessariamente il più appropriato o comunque perfettamente valido anche in altri ordinamenti.

Il punto di vista del progetto di riforma del Model Penal Code sulla mens rea dei reati sessuali "impositivi", la quale dovrebbe sostanziarsi quantomeno nella recklessness, non solo riflette la posizione di autorevoli studiosi, come già accennato, ma è forse anche quello maggioritario. Leggendo i contributi scientifici in materia degli ultimi decenni, si ha l'impressione che la presa di posizione sia in misura significativa condizionata dalla constatazione delle severe conseguenze giuridiche previste solitamente dagli Stati per i sex offenders, per i quali, al di là della pena detentiva - in passato, in alcuni Stati del sud, si è fatto ricorso anche alla pena di morte, in particolare in casi di violenze sessuali nei confronti di persone "bianche" addebitate a imputati afroamericani $^{66}$ - sono previste misure particolarmente stigmatizzanti come l'inclusione (anche a vita) in un registro che può persino essere pubblicamente accessibile, con tanto di fotografie del volto e indicazioni sulla località di residenza ${ }^{67}$. A ciò si collegano ulteriori implicazioni che, almeno nell'ordinamento italiano, evidenzierebbero una difficile compatibilità col principio rieducativo ${ }^{68}$.

Poiché tutte queste severissime conseguenze non sono normalmente sensibili ai profili di colpevolezza e in alcuni casi operano pure a prescindere da essa - così dove è ammessa la strict liability, non rara rispetto alle ipotesi di statutory rape, con l'effetto di precludere ogni mistake-ofage defense ${ }^{69}$ - sembra forte il condizionamento nel senso di argomentare sulla colpevolezza dei reati sessuali a partire da tale quadro, considerandolo quasi un dato immutabile. Ed è qui che si annida quello che sembra essere un limite di prospettiva, che, almeno nell'ordinamento italiano, potrebbe essere evitato senza dover peraltro pensare alle sfumature di decine di giurisdizioni diverse da coordinare. Per quanto suggestivo e in una certa misura comprensibile possa apparire l'argomento basato sulle conseguenze, partire dalla pena per tarare la colpevolezza di un illecito, in particolare de iure condendo, sembra un'inversione metodologica: è la pena, piuttosto, a dover essere influenzata dall'illecito e quindi (anche) dai profili di imputazione colpevole.

Per intendersi, nell'ordinamento italiano, all'introduzione di versioni colpose o parzialmente colpose dei reati sessuali dovrebbe senz'altro accompagnarsi la previsione di risposte sanzionatorie proporzionate, che dovrebbero rendersi tali a partire dai livelli edittali, ma senza trascurare il raccordo con la disciplina dell'art. 609-nonies c.p. ${ }^{70}$, il quale, per quanto non si spinga forse ai livelli di severità delle discipline di common law ${ }^{71}$, contempla comunque delle limitazioni piuttosto serie, che oggi sembrano già applicarsi senza particolari accorgimenti anche ai reati in cui la colpa può porsi come criterio di imputazione effettivo dell'elemento costitutivo dell'età della persona offesa. Il profilo critico di un simile assetto è dato dal fatto che non sempre si tratta di misure che possono essere concretamente disposte in termini proporzionali muovendosi tra un minimo e un massimo: alcune di queste misure hanno carattere perpetuo.

\footnotetext{
${ }^{66}$ Lo testimonia, tra gli altri, M. ViTIELlo, Punishing Sex Offenders, cit., p. 656.

${ }^{67}$ Il Dipartimento di Giustizia degli Stati Uniti mette a disposizione su internet un motore di ricerca che consente di accedere con particolare facilità alle informazioni dei diversi registri statali: www.nsopw.gov .

${ }^{68} \mathrm{Si}$ veda, ad esempio, M. VITIELlo, Punishing Sex Offenders, cit., pp. 667 ss., che descrive il drastico declino della vita quotidiana a cui può andare incontro l'autore di un reato sessuale negli Stati Uniti, potenzialmente penalizzato persino sul piano abitativo, fino a rischiare di ritrovarsi a "vivere" per strada, come testimonierebbero alcune vicende concrete (p. 681).

Sui profili di costituzionalità delle discipline statunitensi, ad esempio, C.L. CARPENTER, A.E. BEVERLIN, The Evolution of Unconstitutionality in Sex Offender Registration Laws, in Hastings Law Journal, 2012, pp. 1071 ss.

${ }^{69}$ Si veda M. VitiEllo, Punishing Sex Offenders, cit., pp. 663-665, 683 e 684, che pone più volte 1'accento sulla sproporzione tra una responsabilità sostanzialmente oggettiva e conseguenze di assoluta severità.

${ }^{70}$ In argomento, ad esempio, S.R. PALUMBIERI, Art. 609 nonies, in A. CAdoPPI, S. CANESTRARI, P. VENEZIANI, Codice penale commentato con dottrina e giurisprudenza, cit., pp. 2590 ss.

${ }^{71}$ Per un confronto, M. BogGIANI, All'origine delle nuove misure introdotte all'art. 609 nonies, $3^{\circ}$ comma, c.p.: le sex offenders laws statunitensi, in Ind. pen., 2012, p. 573 ss.
}

Revista de Direito Brasileira | Florianólopis, SC | v. 25 | n. 10 | p. 280-306 | Jan./Abr. 2020 
Quanto, in particolare, all'assestamento della disciplina modello statunitense sulla recklessness, l'idea che la maggior sensibilità al principio di extrema ratio rimanga più che altro astratta si può coltivare ponendo mente al fatto che il processo sembra esposto alla irresistibile tentazione di considerare effettivamente percepito il rischio di dissenso nella stessa misura in cui l'errore sul medesimo può considerarsi significativamente irragionevole, cioè negli stessi casi in cui il Model Penal Code ritiene sussistente la (criminal) negligence. In altre parole, a parità di rischio elevato, si potrebbe essere indotti a dedurre che l'agente non solo «should be aware» di tale rischio, ma anche la prova del fatto che l'agente, al momento della condotta, «consciously disregards» tale rischio. Pur senza voler avallare questa semplificazione probatoria, sembra però che, aprendo a una colpa grave anche "incosciente", le differenze sarebbero più formali che sostanziali.

Più consistente, invece, è l'osservazione per cui il riferimento solo a canoni di ragionevolezza e non anche a un rischio elevato comporti lo scivolamento verso standard più bassi di quelli della criminal negligence ${ }^{72}$, ma la preferenza per una colpa che, per quanto anche incosciente, sia comunque grave, presidiata da una risposta sanzionatoria proporzionata ${ }^{73}$, dovrebbe consentire di superare buona parte delle perplessità. Così, almeno, dovrebbe essere in un ordinamento come il nostro, dove l'emancipazione da concezioni della colpevolezza ancorate necessariamente a un dato psicologico effettivo è piuttosto assodata.

Inoltre, se si volessero assecondare quelle proposte di riforma che prevedono una pluralità di reati sessuali (a vario titolo) "impositivi", si potrebbe eventualmente pensare di estendere l'area della punibilità per colpa grave soltanto agli illeciti relativi agli atti sessuali più invasivi ${ }^{74}$ : anche questa sarebbe una soluzione di extrema ratio, peraltro in linea con la tradizione italiana che assegna ai comportamenti molesti una natura essenzialmente dolosa, come si è già visto con riferimento alla fattispecie contravvenzionale (quindi potenzialmente aperta anche alla colpa) dell'art. 660 c.p.

\section{BIBLIOGRAFIA}

ACALE SÁnChEZ M., La miopia di 'vostro onore', in Criminal Justice Network, 10 novembre 2019.

AleXAnder D.F., Twenty Years of Morgan: A Criticism of the Subjectivist View of Mens Rea and Rape in Great Britain, in Pace International Law Review, 1995, pp. 207 ss.

\section{AMATI E., L'enigma penale. L'affermazione politica dei populismi nelle democrazie liberali,} Torino, Giappichelli, 2020.

\footnotetext{
${ }^{72}$ M. VitiELLO, Punishing Sex Offenders, cit., pp. 663, 664, 679 e 683. Sulla estrema delicatezza del giudizio di (mera) ragionevolezza rimesso a giurie che sono selezionate dalle parti secondo criteri di strumentalità, per tutti, S.J. SCHULHOFER, Unwanted Sex, cit., pp. 258 e 259: «The reasonableness standard produces a sound result only if the jury itself is reasonable-which is to say, "like us." If it isn't, the reasonableness standard simply invites the worst abuses of cultural stereotyping and ingrained sex bias that rape reformers have tried for so long to escape» (p. 259).

${ }^{73}$ Anche nell'ambito di prospettive femministe non si è mancato di riconoscere l'opportunità di punire comunque di meno gli autori di atti sessuali in colpa rispetto alla percezione del dissenso della persona coinvolta: «My view is that such a "negligent rapist" should be punished, albeit-as in murder-less severely than the man who acts with purpose or knowledge, or even knowledge of the risk» (S. ESTRICH, Rape, in The Yale Law Journal, 1986, p. 1102). Si veda, inoltre, per un'analoga presa di posizione sulla proporzione sanzionatoria, A.E. TASLITZ, Willfully Blinded, cit., pp. 386 e 387.

${ }^{74}$ In senso diverso, A. CADOPPI, M. VITIELlo, A Kiss is Just a Kiss, or is It? A Comparative Look at Italian and American Sex Crimes, in Seton Hall Law Review, 2010, pp. 223, in cui, con particolare riferimento alle prospettive di riforma dell'ordinamento italiano, si propone di riservare la responsabilità colposa solo ai fatti meno gravi.
}

Revista de Direito Brasileira | Florianólopis, SC | v. 25 | n. 10 | p. 280-306 | Jan./Abr. 2020 
Anastasia S., Anselmi M., Falcinelli D., Populismo penale: una prospettiva italiana, $2^{\mathrm{a}}$ ed., Milano, Wolters Kluwer, 2020.

Andersson U., EdGREn M., KARLSSON L., Nilsson G. (edited by), Rape Narratives in Motion, Cham, Palgrave Macmillan, 2019.

BALBI G., I reati contro la libertà e l'autodeterminazione sessuale in una prospettiva di riforma, in Sist. pen., 3 marzo 2020.

BERTOLino M., Libertà sessuale e tutela penale, Milano, Giuffrè, 1993.

Bertolino M., La riforma dei reati di violenza sessuale, in Studium iuris, 1996, pp. 401 ss.

Bertolino M. (a cura di), Verso la riforma dei reati sessuali. Parte I: I reati stricto sensu sessuali, in www.aipdp.it (2018), pp. 2 ss.

BobBio N., voce Libertà, in Enciclopedia del Novecento, Roma, Treccani, 1978, (ora) in www.treccani.it.

Boggiani M., All'origine delle nuove misure introdotte all'art. 609 nonies, $3^{\circ}$ comma, c.p.: le sex offenders laws statunitensi, in Ind. pen., 2012, pp. 573 ss.

BRICOLA F., Funzione promozionale, tecnica premiale e diritto penale, in Quest. crim., 1981, pp. 445 ss.

CADOPPI A., Il reato omissivo proprio, I: profili introduttivi e politico criminali, Padova, Cedam, 1988.

CADOPPI A., Il reato omissivo proprio, II: profili dogmatici, comparatistici e de lege ferenda, Padova, Cedam, 1988.

CAdOPPI A., Art. 609-bis c.p., in ID. (a cura di), Commentario delle norme contro la violenza sessuale e contro la pedofilia, $4^{\mathrm{a}}$ ed., Padova, Cedam, 2006, pp. 439 ss.

CAdoppi A., Il delitto di violenza sessuale (art. 609-bis c.p.), in CADOPPI A., VenEZIANi P., Elementi di diritto penale. Parte speciale. Vol. II. Tomo I, $2^{\text {a }}$ ed., Milano, Wolters KluwerCedam, 2017,pp. 9 ss.

Cadoppi A., Vitiello M., A Kiss is Just a Kiss, or is It? A Comparative Look at Italian and American Sex Crimes, in Seton Hall Law Review, 2010, pp. 191 ss.

CALETti G.M., Libertà e riservatezza sessuale all'epoca di Internet. L'art. 612-ter c.p.e l'incriminazione della pornografia non consensuale, in Riv. it. dir. proc. pen., 2019, pp. 2045 ss.

CARD R., GillesPie A.A., Hirst M., Sexual Offences, Bristol, Jordans, 2008. 
CARPEnTER C.L., Beverlin A.E., The Evolution of Unconstitutionality in Sex Offender Registration Laws, in Hastings Law Journal, 2012, pp. 1071 ss.

Coy M., Kelly L., VerA-Gray F., GARner M., KANYEREDZI A., From 'no means no' to 'an enthusiastic yes': changing the discourse on sexual consent through Sex and Relationships Education, in Sundaram V., SAUNDERS H. (edited by), Global Perspectives and Debates on Sex and Relationships Education. Addressing Issues of Gender, Sexuality, Plurality and Power, London, Palgrave Macmillan, 2016, pp. 84 ss.

De Simone G., voce Violenza, in Enc. dir., XLVI, Milano, Giuffrè, 1993, pp. 881 ss.

Donini M., Populismo e ragione pubblica. Il post-illuminismo penale tra lex e ius, Modena, Mucchi Editore, 2019.

DRESSLER J., Understanding Criminal Law, 8ª ed., Durham, Carolina Academic Press, 2018.

Dressler J., Garvey S.P., Criminal Law. Cases and Materials, $7^{\text {th }}$ ed., Saint Paul, West Academic Publishing, 2016.

Duncan M.J., Sex Crimes and Sexual Miscues: The Need for a Clearer Line Between Forcible Rape and Nonconsensual Sex, in Wake Forest Law Review, 2007, pp. 1087 ss.

EISELE J., § 177, in Schönke/Schröder Strafrechtgesetzbuch Kommentar, 30a ed., München, Beck, 2019, pp. 1792 ss.

ESTRICH S., Rape, in The Yale Law Journal, 1986, pp. 1087 ss.

FARALDO CABANA P., ¿Intimidación o prevalimiento? La sentencia de La Manada y los delitos sexuales en España, in Criminal Justice Network, 25 settembre 2018.

Faraldo Cabana P., Alcalé Sánchez M. (directoras); RodríGuez LóPez S., Fuentes LOUREIRO M.A. (coordinadoras), La Manada. Un antes y un después en la regulación de los delitos sexuales en España, Valencia, Tirant lo Blanch, 2018.

FERRAJOli L., Il populismo penale nell'età dei populismi politici, in Questione giustizia, 1/2019, pp. 79 ss.

FERZAN K.K., Consent, Culpability, and the Law of Rape, in Ohio State Journal of Criminal Law, 2016, pp. 397 ss.

FIANDACA G., Luci ed ombre della pronuncia a sezioni unite sul trattamento medico-chirurgico arbitrario, in Foro it., 2009, II, cc. 306 ss.

FIANDaCa G., Populismo politico e populismo giudiziario, in Criminalia, 2013, pp. 95 ss. 
Fradella H.F., FAHMy C., Rape and Related Offenses, in Fradella H.F., Sumner J.M. (edited by), Sex, Sexuality, Law, and (In)Justice, New York, Routledge, 2016, pp. 138 ss.

FRANCOLINi G., Ai limiti della violenza sessuale: il dibattuto problema della rilevanza penale dei toccamenti repentini ed insidiosi, in Riv. pen., 2006, pp. 716 ss.

Garcia A., Consentimiento. Una nueva y polémica sentencia obliga a revisar el delito de violación, in https://elpais.com, 2 novembre 2019.

GatTa G.L., La minaccia. Contributo allo studio delle modalità della condotta penalmente rilevante, Roma, Aracne, 2013.

GatTa G.L., Dalle Sezioni Unite il criterio per distinguere concussione e 'induzione indebita': minaccia di un danno ingiusto vs. prospettazione di un vantaggio indebito, in Dir. pen. cont., 17 marzo 2014.

Gatta G.L., Atti sessuali su minore incosciente per effetto dell'uso di alcool e droga: violenza o abuso sessuale? Il caso spagnolo della 'Manada de Manresa', nella prospettiva del penalista italiano. Sui confini dei concetti di violenza e minaccia, in Criminal Justice Network, 10 novembre 2019.

Gerstmann E., Campus Sexual Assault. Constitutional Rights and Fundamental Fairness, Cambridge, Cambridge University Press, 2019.

GoIsIS L., La violenza sessuale: profili storici e criminologici. Una storia 'di genere', in Dir. pen. cont., 31 ottobre 2012.

Herring J., Criminal Law, 10 ${ }^{\text {th }}$ ed., London, Palgrave, 2017.

Hofverberg E., Sweden: Supreme Court Defines Negligent Rape, in Global Legal Monitor, 17 luglio 2019.

HöRnLE T., The New German Law on Sexual Assault and Sexual Harassment, in German Law Journal, 2017, pp. 1309 ss.

HöRNLE T., \#MeToo - Implications for Criminal Law?, in Bergen Journal of Criminal Law and Criminal Justice, 2018, pp. 115 ss.

HuSAK D., The Philosophy of Criminal Law. Selected Essays, Oxford, Oxford University Press, 2010.

INSOLERA G., Il populismo penale, in disCrimen, 13 giugno 2019.

LASCURAÍn SÁNCHEZ J.A., ¿Qué es una violación?, in Criminal Justice Network, 2 ottobre 2018. 
LASCURAín SÁNCHEZ J.A., La sentencia de La Manada: ¿todos contentos?, in Almacén de derecho, 4 novembre 2019.

LASCURAÍn SÁnCHEZ J.A., Delitos sexuales: ¿una reforma progresista?, in Almacén de derecho, 28 marzo 2020.

Le Goaziou V., Viol. Que fait la justice?, Paris, Sciences Po, 2019.

LEGrand C.E., Rape and Rape Laws: Sexism in Society and Law, in California Law Review, 1973, pp. 919 ss.

MacKinnon C.A., Sexual Harassment of Working Women. A Case of Sex Discrimination, Hew Haven, London, Yale University Press, 1979.

MacKInnon C.A., Feminism, Marxism, Method, and the State: Toward Feminist Jurisprudence, in Signs, 1983, pp. 635 ss.

MACRÌ F., Verso un nuovo diritto penale sessuale. Diritto vivente, diritto comparato e prospettive di riforma, Firenze, Firenze University Press, 2010.

MACRÌ F., La riforma dei reati sessuali in Germania. Centralità del dissenso e "tolleranza zero" verso le molestie sessuali tra diritto penale simbolico e potenziamento effettivo della tutela della sfera sessuale, in Dir. pen. cont., 24 novembre 2016.

Mantovani M., voce Violenza privata, in Enc. dir., XLVI, Milano, Giuffrè, 1993, pp. 930 ss.

MAZZACUVA F., La violenza sessuale di gruppo (art. 609-octies c.p.), in CADOPPI A., VENEZIANI P., Elementi di diritto penale. Parte speciale. Vol. II. Tomo I, $2^{\text {a }}$ ed., Milano, Wolters KluwerCedam, 2017, pp. 54 ss.

Palumbieri S.R., Art. 609 bis, in CAdoppi A., CAnestrari S., Veneziani P., Codice penale commentato con dottrina e giurisprudenza, Torino, Giappichelli, 2018, pp. 2523 ss.

Palumbieri S.R., Art. 609 nonies, in CAdoppi A., CANeStrari S., Veneziani P., Codice penale commentato con dottrina e giurisprudenza, Torino, Giappichelli, 2018, pp. 2590 ss.

PAPA M., Quando c'è l'ebrezza il consenso della vittima è sempre anomalo: i limiti delle nostre leggi, in www.repubblica.it, 15 settembre 2017.

PAPA M., La fisiognomica della condotta illecita nella struttura dei reati sessuali: appunti per una riflessione sulla crisi della tipicità, in Criminalia, 2018, pp. 213 ss.

PARMIGGIANI M.C., Rape by omission, ovvero lo "stupro omissivo": note a margine di un recente caso californiano, in Ind. pen., 2005, pp. 311 ss. 
PÉREZ Alonso E., Concepto de abuso sexual: contenido y límite mínimo del delito de abusos sexuales, in InDret, 3/2019.

PISA P., Le nuove norme contro la violenza sessuale. Il commento, in Dir. pen. proc., 1996, pp. 285 ss.

PORGES S.W., La teoria polivagale. Fondamenti neurofisiologici delle emozioni, dell'attaccamento, della comunicazione e dell'autoregolazione, Roma, Fioriti, 2014.

PRESNO LINERA M.A., ¿Son legítimas las sentencias juzgadas socialmente como injustas?, in Almacén de derecho, 6 novembre 2018.

PUlitanò D., Populismi e penale. Sulla attuale situazione spirituale della giustizia penale, in Criminalia, 2013, pp. 123 ss.

RoACH K., Criminal Law, $7^{\text {th }}$ ed., Toronto, Irwin Law, 2018.

Romano B., Proposte di riforma nei delitti contro la sfera sessuale della persona, in Dir. pen. cont., 29 novembre 2018.

RoOK P., WARD R., Sexual Offences. Law \& Practice, 5 ${ }^{\text {th }}$ ed., London, Sweet \& Maxwell, 2016.

Sabadell A.L., Forme di “patriarcalismo" giuridico-penale in Brasile. Il caso delle molestie sessuali e della violenza sessuale, in Criminalia, 2016, pp. 563 ss.

SCHUlHofer S.J., Taking Sexual Autonomy Seriously: Rape Law and beyond, in Law and Philosophy, 1992, pp. 35 ss.

SCHUlHOFER S.J., The Feminist Challenge in Criminal Law, in University of Pennsylvania Law Review, 1995, pp. 2151 ss.

Schulhofer S.J., Unwanted Sex. The Culture of Intimidation and the Failure of the Law, Cambridge, London, Harvard University Press, 1998.

SCHULHOFER S.J., Reforming the Law of Rape, in Law \& Inequality: A Journal of Theory and Practice, 2017, pp. 335 ss.

SPENA A., Harmless Rapes? A False Problem for the Harm Principle, in Dir. \& quest. pubb., 2010, pp. 497 ss.

Stevenson K., Davies A., Gunn M., Blackstone's Guide to The Sexual Offences Act 2003, Oxford, Oxford University Press, 2004.

SzEGÖ A., Quando lo stupro è legale: la "marital rape exemption”, in Riv. it. dir. proc. pen., 1995, pp. 853 ss. 
TASLITZ A.E., Willfully Blinded: On Date Rape and Self-Deception, in Harvard Journal of Law \& Gender, 2005, pp. 381 ss.

The American Law Institute, Model Penal Code: Sexual Assault and Related Offenses, Tentative Draft No. 3 (April 6, 2017), Philadelphia, The American Law Institute, 2017.

TORDini Cagli S., Profili penali del trattamento medico-chirurgico in assenza di consenso, in Resp. civ. prev., 2009, pp. 1060 ss.

VIGANÒ F., La tutela penale della libertà individuale, I, L'offesa mediante violenza, Milano, Giuffrè, 2002.

VIGANÒ F., Omessa acquisizione del consenso informato del paziente e responsabilità penale del chirurgo: l'approdo (provvisorio?) delle Sezioni unite, in Cass. pen., 2009, pp. 1811 ss.

VIRGILIo M., Violenza sessuale e norma. Legislazioni penali a confronto, Ancona, Nuove Ricerche, 1996.

VitiEllo M., Punishing Sex Offenders: When Good Intentions Go Bad, in Arizona State Law Journal, 2008, pp. 651 ss.

Vogliotti M., Dove passa il confine? Sul divieto di analogia nel diritto penale, Torino, Giappichelli, 2011. 